\title{
Understanding the response of the ionosphere-magnetosphere system to sudden solar wind density increases
}

\author{
Yi-Qun $\mathrm{Yu}^{1}$ and Aaron J. Ridley ${ }^{1}$ \\ Received 27 June 2010; revised 20 January 2011; accepted 1 February 2011; published 15 April 2011.
}

[1] The different responses of the magnetosphere to sudden solar wind density enhancements are investigated. The majority of the responses are observed to have two phases: the first is due to an inductive $E$ field propagating through the system, while the second is due to a vortex formation in the magnetosphere. However, during some events, the system displays a single-phase response. In order to understand the controlling parameters in the system response, statistical analyses of several solar wind external characteristics as well as the internal index $D_{s t}$ are conducted. Results of these analyses show that none of those characteristics seem to be associated with the way the geospace system responds to the abrupt solar wind density increase. Owing to the lack of statistics in the analysis, simulations investigating the effect of the solar wind Alfvén Mach number on the magnetospheric response have been conducted. The simulation results indicate that the two-phase response is always present but that a low solar wind Mach number causes an indistinct two-phase response, which is difficult to identify as having two phases. Compression signals following the sudden compression at the magnetopause are carried by a fast mode wave in the magnetosphere, which propagates toward the Earth and is reflected back toward the magnetopause by the plasmapause or ionosphere. The wave is subsequently reflected back again, leading to a secondary Earthward propagation. These multiple bounces of the fast mode wave result in two groups of the two-phase responses in the ionosphere, although the second group is extremely difficult to identify in ionospheric data.

Citation: Yu, Y.-Q., and A. J. Ridley (2011), Understanding the response of the ionosphere-magnetosphere system to sudden solar wind density increases, J. Geophys. Res., 116, A04210, doi:10.1029/2010JA015871.

\section{Introduction}

[2] The effects of solar wind dynamic pressure enhancements on the dynamics of the Earth's ionosphere-magnetosphere system have been studied for many years, primarily utilizing two observational techniques (ground-based magnetometer observations and SuperDARN observations):

[3] 1. Ground-based magnetometer observations indicate a two-phase response after a sudden dynamic pressure enhancement [e.g., Friis-Christensen et al., 1988; Farrugia et al., 1989; Russell and Ginskey, 1995; Moretto et al., 2000; Sibeck et al., 2003]. The response is observed over a large range of latitudes. At higher latitudes (polar cap latitude) on the morning side, the first response shows a negative pulse in the $\mathrm{H}$ component of the magnetic perturbation and the second response indicates a positive deflection. At lower latitudes, the $\mathrm{H}$ component shows a positive pulse in the first response and a negative deflection in the second. On the afternoon side, this bipolar change of the magnetic perturbation behaves in the opposite manner.

\footnotetext{
${ }^{1}$ Center for Space Environment Modeling, University of Michigan, Ann Arbor, Michigan, USA.

Copyright 2011 by the American Geophysical Union. 0148-0227/11/2010JA015871
}

[4] 2. SuperDARN measurements (and other techniques revealing the ionospheric potential) show that a pair of convection vortices appears in the dayside ionosphere after a sudden dynamic pressure enhancement encounters the magnetosphere, and a second pair of vortices follows [e.g., Engebretson et al., 1999; Boudouridis et al., 2008]. The first pair of vortices rotates clockwise on the morning side and counter clockwise on the afternoon side. The second pair behaves in an opposite manner. The plasma convection flows in a direction opposite to the ionospheric Hall current, which is the major contributor to the ground-based magnetic perturbation at high latitudes. The convection has a consistent behavior with the previously mentioned bipolar signature observed in the magnetic perturbations.

[5] Both types of observations (i.e., ground-based magnetometer and SuperDARN) indicate that a sudden solar wind dynamic pressure increase that impacts on the magnetosphere results in responses that occur in two phases in the ionosphere. These responses have been reproduced by a wide variety of simulation studies [e.g., Lysak and Lee, 1992; Slinker et al., 1999; Fujita et al., 2003a, 2003b; Keller et al., 2002; Yu and Ridley, 2009]. Besides the above ionospheric and ground-based responses to sudden increases in the solar wind dynamic pressure, studies focusing on responses in other geospace regions utilizing other techniques have also 
been extensively conducted. For example, Zong et al. [2009] investigated the energetic electrons in the outer radiation belt after an interplanetary shock. Zong et al. [2010] study middle- and low-latitude dayside ionospheric characteristics after two passages of interplanetary shocks using the digisonde global ionospheric radio observatory (GIRO). While various important responses throughout the geospace system are being explored, this paper particularly focuses on the two-phase responses.

[6] A significant amount of work has focused on understanding the generation mechanism of the two-phase responses and many of these studies have found that the dynamics of field-aligned currents (FACs) are responsible for the two-phase responses. FACs flow into and out of the ionosphere, producing convection vortices there. Consequently, among past studies, the main argument is on how FACs are generated after the sudden solar wind dynamic pressure increase encounters the magnetosphere and how they subsequently influence the ionospheric response. Kivelson and Southwood [1991] proposed a model suggesting that FACs are directly generated by the shear Alfvén perturbations at the low-latitude boundary layer (LLBL). Tamao [1964a, 1964b] and Southwood and Kivelson [1990] suggested that the development of vortical flows in the ionosphere results from the conversion of compressional waves to transverse waves, which carries FACs into the ionosphere. However, these studies did not consider the two responses separately.

[7] In contrast, individual treatment of the two responses were reported by Araki [1994], Engebretson et al. [1999], Moretto et al. [2000], Keller et al. [2002], Fujita et al. [2003a, 2003b], Stauning and Troshichev [2008], Yu and Ridley [2009], and Samsonov et al. [2010]. Araki [1994] proposed a physical model in which the first response results from the dusk-to-dawn inductive electric field caused by the compressional fast mode wave, and the second response is a result of enhanced convection electric fields in the new compressed configuration. Keller et al. [2002] suggested that the first response is due to the current generated by Alfvén waves that are driven by dynamic pressure perturbations near the magnetopause and that the second response is due to shear Alfvén waves. Fujita et al. [2003a, 2003b] conducted an MHD simulation with a sudden density increase in the solar wind. The authors concluded that the current system associated with the first response is initially generated at the magnetopause and subsequently is converted to FACs through a mode wave conversion between the compressional fast mode wave and the Alfvén wave. The authors also concluded that the current system, which is responsible for the second response, is driven by a dynamo in the tailward area of the cusp region. Stauning and Troshichev [2008] suggested that the first pair of FACs is associated with excess magnetopause boundary currents, which may be diverted along cusp field lines to close at the ionospheric foot points, while the second pair of FACs is related to temporarily increased Region-1 FACs. A recent study by Samsonov et al. [2010] explored the sources in the magnetosphere for the two pairs of ionospheric currents using a global MHD simulation under northward interplanetary magnetic field (IMF) conditions. They found that the first transient response is connected to energy dynamo from the shock-intensified lobe reconnection, while the second transient response is related to the dynamo near the equatorial plane at the flanks which is associated with the reflected fast shock inside the magnetosphere. Yu and Ridley [2009] numerically studied the two responses with different IMF orientations. The authors found that the first response is likely caused by the same generation mechanism under both northward and southward IMF conditions. The sudden compression of the magnetosphere, the starting point of the generation mechanism, results in fast mode wave propagation through the dayside magnetosphere, inducing a dusk-todawn electric field. Subsequently, the dusk-to-dawn inductive currents turn into FACs at high latitude near the dayside cusp region. The FACs flow into the ionosphere on the afternoon side and out of the ionosphere on the morning side. With regard to the second response, they proposed that FACs are generated differently under different IMF conditions: In a northward IMF situation, FACs are generated from magnetospheric vortices, which are caused by thermal pressure gradients inside the magnetosphere after the high solar wind dynamic pressure impacts on the magnetosphere. Whereas in the southward IMF case, no thermal pressure gradient is observed inside the magnetosphere; however plasma shear flow in the dayside magnetopause produces convection vortices and thus FACs.

[8] In order to have a clear reference to the first and second responses, they will be referred to as "Ey-response" and "Vortex-response," respectively, in the rest of the paper. These names are directly related to their generation mechanisms and so self-described.

[9] With a large number of simulations and observation reports available on the responses of the geospace system to a discontinuity in the solar wind density, few studies have investigated the two individual responses statistically. In this paper, density increase events from 1996-2002 are studied. The selection of the events follows the criterion: $\frac{d N}{N}>2$ within $3 \mathrm{~min}$, where $d N$ is the change of solar wind number density $(N)$ across the discontinuity. The responses observed from the Assimilative Modeling of Ionospheric Electrodynamics (AMIE), Richmond and Kamide [1988] technique, geosynchronous satellites, and ground-based magnetometers are examined. It is found that not all the events respond in the "typical" two-phase manner to the sudden density increase. To further understand the categories sorted out from the observations, statistical analyses on various external solar wind characteristics as well as simulations are conducted. Section 2 describes the observational study of the sudden dynamic pressure enhancement and the corresponding phenomena observed by various measurement methods; section 3 presents simulation results, investigating the influence of the solar wind Alfvén Mach number on the responses and its implications; and section 4 focuses on the wave propagation after the sudden compression of the magnetosphere and its influence on the ionospheric responses.

\section{Observations}

[10] As shown in the simulation from Yu and Ridley [2009], it is possible to use the cross polar cap potential (CPCP) index as a reference in determining whether two responses are observed after a sudden dynamic pressure enhancement encounters the magnetosphere. AMIE provides $1 \mathrm{~min}$ resolution data, from which the CPCP index can easily be obtained [Ridley and Kihn, 2004]. However, as the CPCP 
index obtained from AMIE is the difference between the maximum and minimum electric potentials in the ionosphere, the development of the CPCP might not thoroughly reflect the ionospheric vortices that appear locally. Therefore, other selection criteria such as ground-based magnetic perturbations from dayside magnetometer stations, potential patterns driven by AMIE over the global region, and Polar Cap North (PCN) index measured by one northern hemisphere high-latitude magnetometer are used to further identify the occurrence of the two-phase responses. As is mentioned in the Introduction, for a typical two-phase response, the ground-based observation of perturbations should show bipolar variations, and the AMIE potential patterns should display two pairs of potential cells (convection vortices) for the two-phase responses. Furthermore, the PC index should first indicate a negative pulse in the Ey-response and then a positive increase in the Vortex-response [Lukianova, 2003]. These criteria help to categorize the interesting events into two groups as discussed below.

[11] When very little response to an event is observed within the database, that event is excluded. The lack of response could be due to the solar wind feature missing the magnetosphere, or some other reason, outside the scope of this research study. For all events in which a clear response was observed, we classify the density increase events into two basic groups. Those events with responses in two phases are categorized into "two-phase response," (i.e, the responses throughout the geospace system are represented by bipolar ground-based magnetic field perturbations, two successive pairs of potential cells with opposite polarity, and bipolar pulses in the PCN index), while those with a single phase response (either acting as the Vortex-response or the other) are classified into "single-phase response" (i.e., the responses do not show bipolar but unipolar variations). Figures 1, 2, 3, 4,5 , and 6 show two examples that demonstrate the "twophase" group (Figures 1-3) and the "single-phase" group (Figures 4-6).

\subsection{Two Observational Examples}

[12] The first example, illustrated in Figures 1-3, shows responses observed from the ionosphere and the ground. At 14:01 UT on 4 January 2001 (denoted by the dashed line), the OMNI data (Figure 1) indicates a sudden solar wind density increase under northward IMF encountering the magnetopause. After this solar wind density increase, the PC index (the fifth line plot) shows a negative pulse before the index increases significantly and the CPCP index (the last line plot) obtained from AMIE depicts two successive peaks. The magnetometer observations (Figure 2a), which are obtained from ground magnetometer stations located above $50^{\circ}$ magnetic latitude on the morning side, display bipolar perturbations. Potential patterns (Figure 3) from AMIE also show two responses: the first pair of potential cells appears at 14:02 UT around $70^{\circ}$ magnetic latitude on the dayside ionosphere, and the second pair with opposite polarity appears after about 2 min.

[13] In addition to the ionospheric and ground responses that are described above, the magnetospheric responses are examined. To do so, geosynchronous data from GOES satellites (Figure 2b) are utilized. The observed magnetic fields by GOES 8 and 10 on the dawn side show that the $\mathrm{Bz}$ component increases after the sudden compression of the magnetosphere. In conclusion, all the above observations indicate that the sudden solar wind density increase that impacts the magnetosphere causes a wide range of perturbation throughout geospace.

[14] However, some of these events involved with sudden solar wind density enhancements do not show definitive twophase responses. One such "single-phase" example is shown in Figures 4-6, which utilizes the same database as in Figures 1-3. From the OMNI 1 min data, an increase of solar wind was observed at 16:46 UT on 11 May 2002 when the IMF Bz was southward. However, the ground-based perturbations in the postnoon sector did not display clear two-phase responses from high latitudes to low latitudes, and AMIE output of the residual potential pattern did not show two successive pairs of potential cells emerging from the dayside ionosphere, although a disturbance in the geosynchronous orbit was observed. The disturbance in the ionosphere appears to be more like the second phase (or Vortexresponse) of the typical two-phase responses, but the first phase (or Ey-response) is not observed in this event. As the $D_{s t}$ index indicates a highly disturbed system and substorm activity (by checking AL index) indeed occurred preceded this example event, the nightside in the ionospheric potential pattern shows significant disturbances.

\subsection{Statistical View}

[15] The CPCP index obtained from simulation results [ $\mathrm{Yu}$ and Ridley, 2009] shows different behavior for different IMF orientations. Under northward IMF conditions, there are two peaks in the CPCP profile. While under the southward IMF conditions, the index decreases first before increasing to a peak. To find the same phenomena from the observational point of view, OMNI data and AMIE data are utilized. Twenty-three events of a solar wind density increase, mostly with continuously northward IMF for at least $30 \mathrm{~min}$, and 41 events, mostly with continuously southward IMF for at least $30 \mathrm{~min}$, were found and selected to make epoch plots, which are shown in Figure 7. The epoch time $t=0$ is the time when the ionosphere starts to react to the sudden increase of the solar wind density. Two peaks are barely observed in the AMIE CPCP profile after the epoch time in the northward case; while in the southward situation, the CPCP slightly decreases and is followed by an increase. These behaviors are consistent with the simulation results by $Y u$ and Ridley [2009], although they are more subtle in the epoch plots. The subtleness in the plots could be a result of slight timing difference in different events and the fact that the time scale of the features are a few minutes, while the temporal resolution of the AMIE CPCP is $1 \mathrm{~min}$. This means that there are only a few measurements across each event, so it is easy to miss the main maxima and minima. Further, the AMIE technique utilizes a nonuniform distribution of data, such that, for each event, the magnetometers may not be in the optimum positions to measure the global distribution, and therefore the CPCP may have errors. Nevertheless, the features are subtle, but visible.

[16] While these events show typical two-phase responses ("two-phase" group), there are events that do not show the first response (or Ey-response) ("single-phase" group), as previously illustrated from the second example in Figures 4 6 . One attempt to explain the difference was first described by Lukianova [2003], who studied the first response by using the 


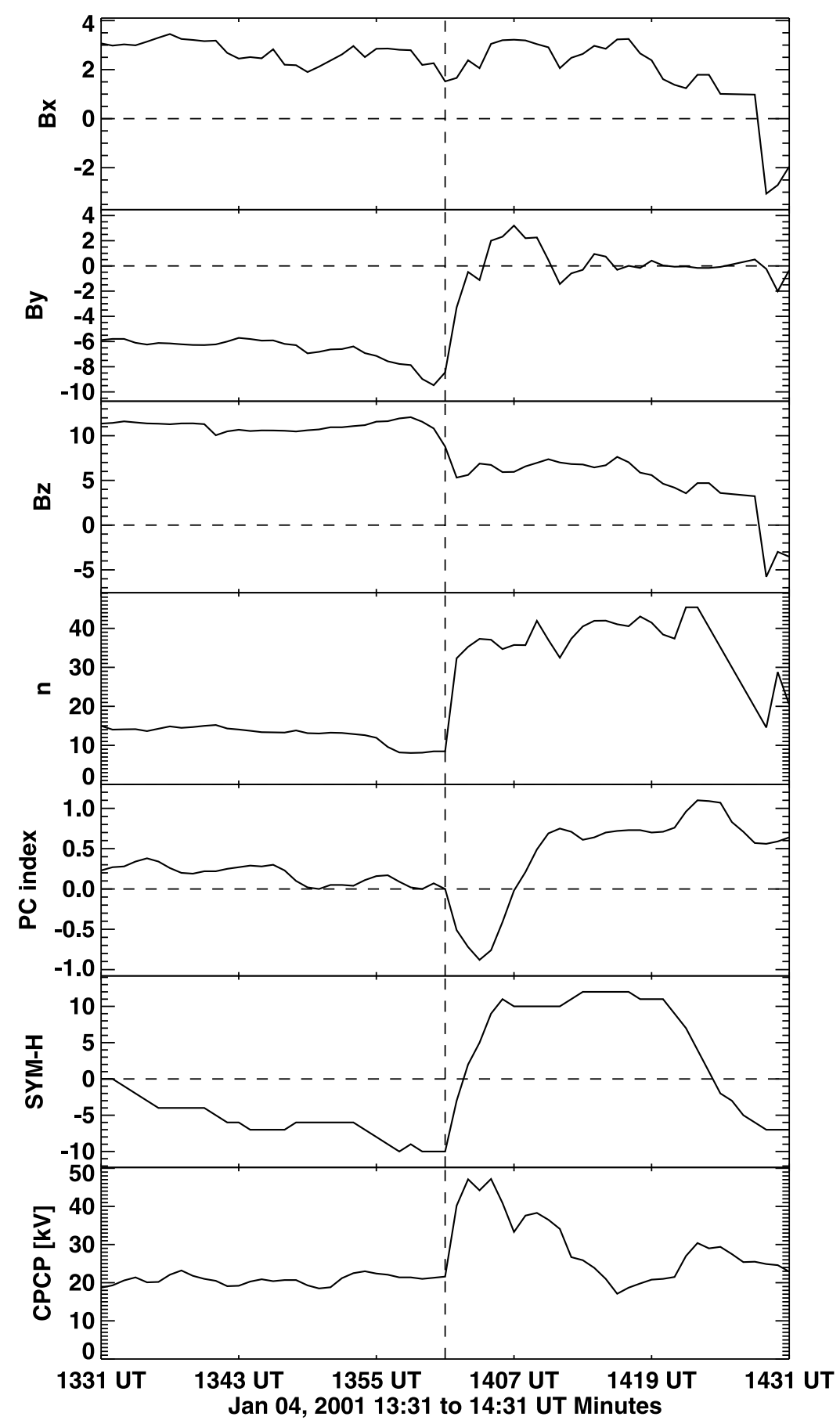

Figure 1. One example from the "two-phase" group of the responses observed from the ground, the ionosphere, and the magnetosphere due to the sudden solar wind density increase on 4 January 2001. Figure 1 lists the IMF $B_{x}, B_{y}, B_{z}$, solar wind density, PC index from ground observations, SYM-H index, and the cross polar cap potential from AMIE. The dashed line indicates the time the solar wind density increases.

PC index and suggested that during magnetic storms, the events tend not to have the first response.

[17] This study, however, could not strongly support or negate the conclusion from Lukianova [2003]. Attempts by using $D_{s t}$ index to understand the two-phase response have also been carried out here. Figure 8 a shows a histogram of the "two-phase" group with respect to $D_{s t}$. Note that most of the events concentrate around small values of the $D_{s t}$ index, but during storm times, some events with a large $D_{s t}$ index can also show two responses. Furthermore, a histogram for the "single-phase" group in the lower plot of Figure 8a shows that the $D_{s t}$ index for the "single-phase" group is not limited to the large values (or storm time), but there are many fewer events in this category and the distribution of these events is very sporadic. Therefore, we cannot conclude such that with a higher storm activity level the events tend to have single- 


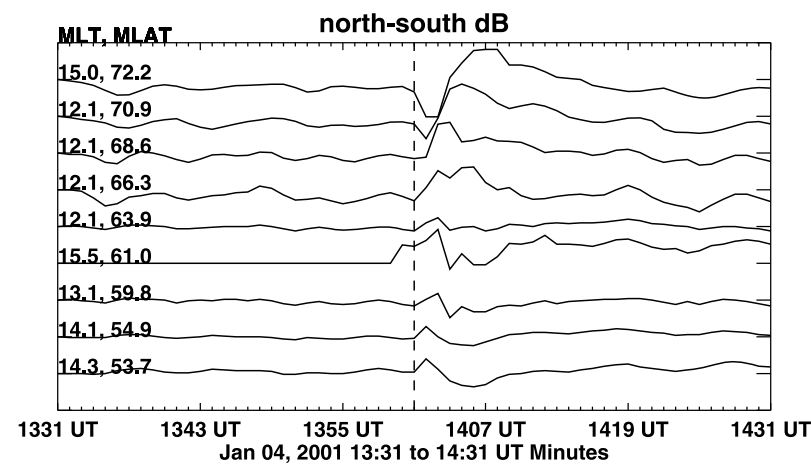

(a) Ground-based data

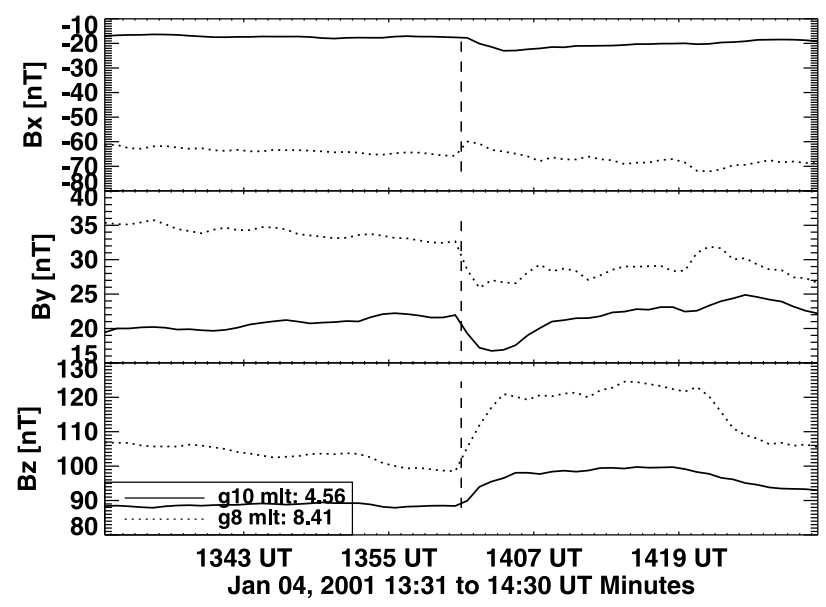

(b) GOES data

Figure 2. For the event on 4 January 2001, (a) the groundbased observations about magnetic perturbations in the morning side are shown above $50^{\circ}$ of magnetic latitude, and the lines are in a order according to the latitudes. The two columns of numbers indicate the magnetic local time (MLT) and magnetic latitude (MLAT) of each station. (b) The magnetospheric disturbance observed from the GOES satellite is shown.

phase response, as suggested by Lukianova [2003]. However, the possibility of the above relationship remains, as will be discussed in below.

[18] Several other attempts are described in this paper in order to determine the controlling factor(s) that make(s) a difference in the way geospace responds to a sudden solar wind density increase. In other words, which factor(s) control(s) whether or not geospace reacts with two-phase responses? The factors that are examined include (1) the net increase of dynamic pressure, (2) the solar wind Alfvén Mach number, (3) the plasma $\beta$ for protons in the solar wind, (4) the rate of solar wind density increase, and (5) the orientation of the IMF. These parameters, representing the external solar wind driving, are chosen from the time just before the highdensity discontinuity in the solar wind encounters the bow shock. Figures $8 \mathrm{~b}-8 \mathrm{f}$ shows histograms of the two groups corresponding to these parameters. It is found that there is no clear separation between the two categories in these parameters. In other words, a pattern such that the two categories prefer to occur in two different ranges of any of the parameters is not observed here, implying that none of these parameters seem to distinctively control the manner the system responds. It is possible that a combination of some of the above external solar wind characteristics controls the way the geospace responds. It is also possible that the geospace system itself, rather than the external drivers, determines the nature of the responses. But, these possibilities still require further investigation.

[19] However, some possible tendencies still exist. For example, for the $\mathrm{Bz}$ orientation (Figure $8 \mathrm{~d}$ ), most of the second category prefer to occur under a southward IMF condition; For the Alfvén Mach number (Figure 8b), the second category events mostly concentrate on the range for a small Alfvén Mach number; such trend is also observed in Figure 8e. Nevertheless, many events from the first category also occur in these ranges, leading to a dilemma that no definitive conclusion could be drawn. One could conclude that with a southward IMF or a more disturbed time period the system is more likely to have a single-phase response, only if there were many fewer two-category events than one-phase events in the same IMF or $D_{s t}$ situation. Apparently, this is not the case here.

[20] One possible explanation for this uncertainty is that the identification of the two-phase response becomes difficult when the system is highly disturbed. As Yu and Ridley [2009] have simulated both southward and northward IMF conditions, and found that the net changes caused by the increase in the solar wind density are the same for both conditions, but they needed to subtract the background electric potential/ FACs in the southward IMF case in order to identify the net changes due to its opposite polarity to the background electric potential/FACs. The situation may be the same here in these observations. Because of the significant background, the changes caused by the solar wind variation can be partly submerged, leading to an integrated responses that are not clear enough to be identified.

[21] One of the difficulties with utilizing observational data is that there are often too few events to have the statistics needed in order to adequately explore the various combinations of drivers that may cause one type of reaction or another. Note that the number of events is highly dependent on the criteria used in the selection. Zhang et al. [2010] utilized a criteria of $\frac{d N}{N}>0.5$ to select the dynamic pressure increase events, instead of using $\frac{d N}{N}>2$ as in this paper; therefore a lot more events were presented in their work. Furthermore, the study in this paper needs to sort out the events that clearly show the two-phase responses, which further reduces the number of events for the statistical study. We therefore turn to simulations to attempt to determine the role of the solar wind Alfvén Mach number on the geospace system response to solar wind density increases. The Mach number is chosen because in the histograms (Figure 8) of the two categories, the events in the second category tend to cluster around the low Mach numbers. The simulation results lead us to believe that the Mach number does indeed play a significant role in the manifestation of the two responses. The results also imply that the typical two-phase responses are always existent, regardless of the magnitude of the Mach number; however, in low Mach number situations, the typical two-phase response, in the form of the CPCP index or the ground magnetic perturbations, are not so distinct as that in high Mach number 
electric potential over electric potential

AMIE Version 2.21

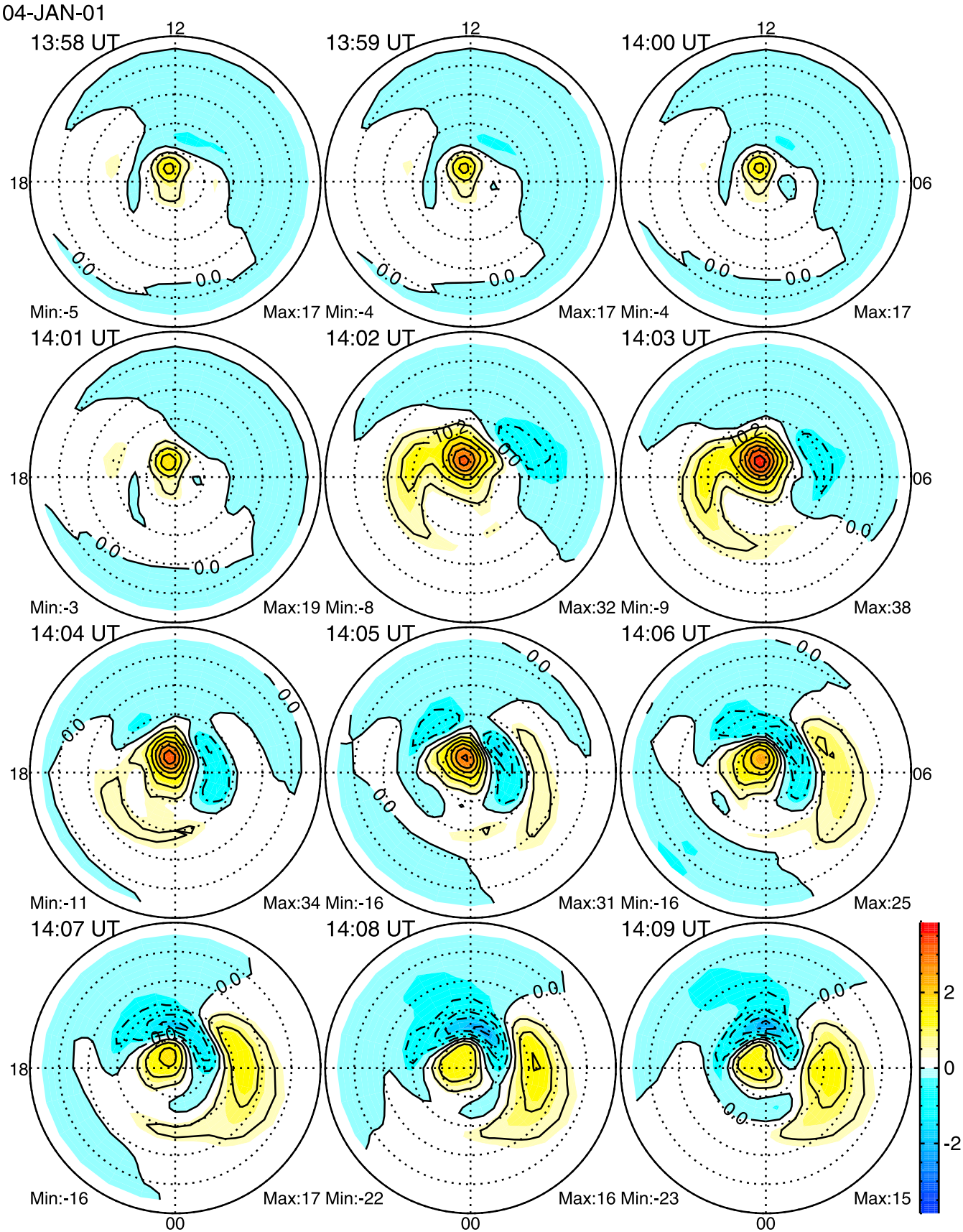

Figure 3. For the event on 4 January 2001, the ionospheric response in the AMIE electric potential pattern. Yellow indicates positive potential, and blue means negative potential. 


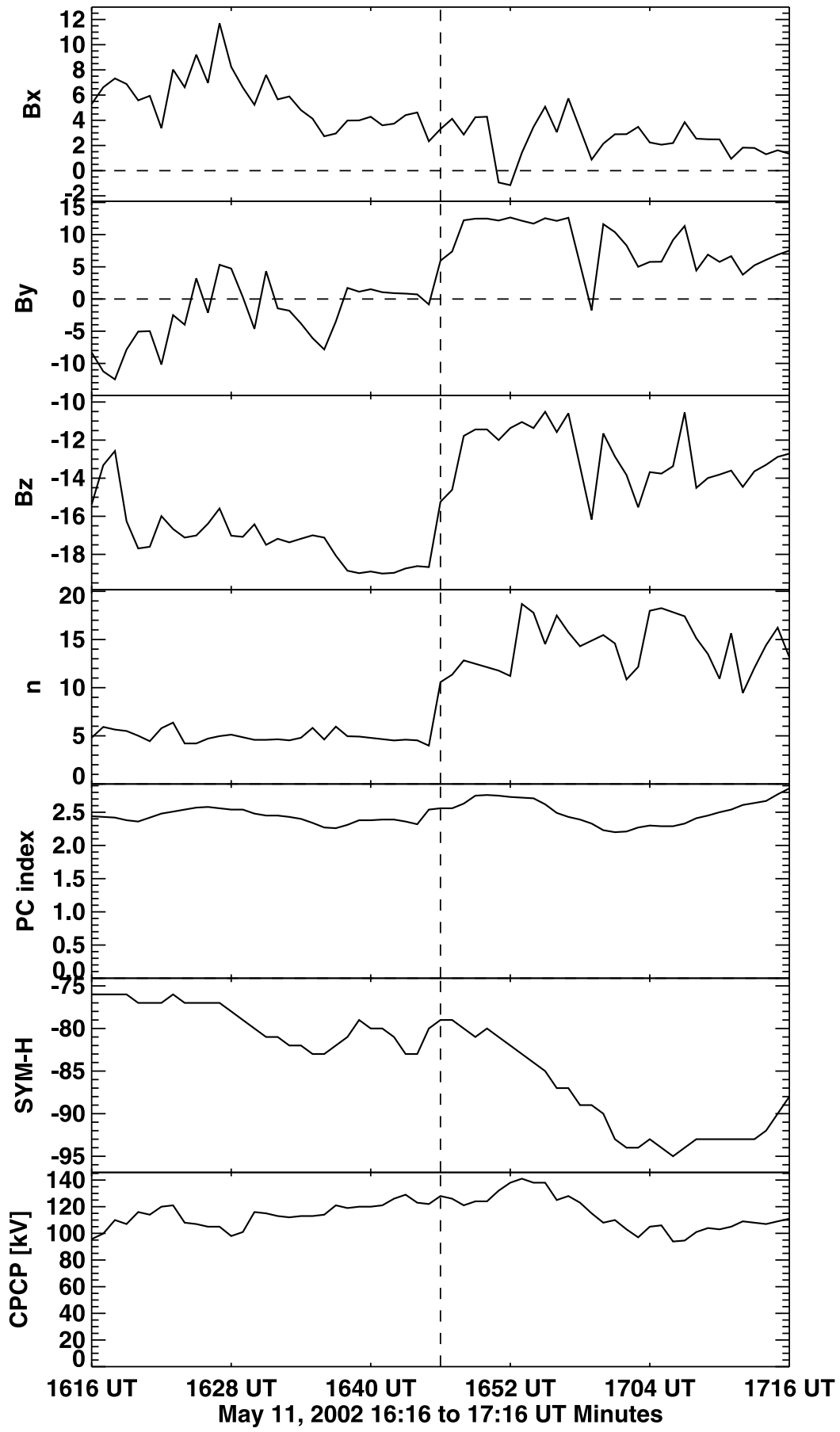

Figure 4. One example from "single-phase" group of disturbances observed from the ground, the ionosphere, and the magnetosphere due to the sudden solar wind density increase at 16:46 11 May 2002. Figure 4 is in the same format as Figure 1. 


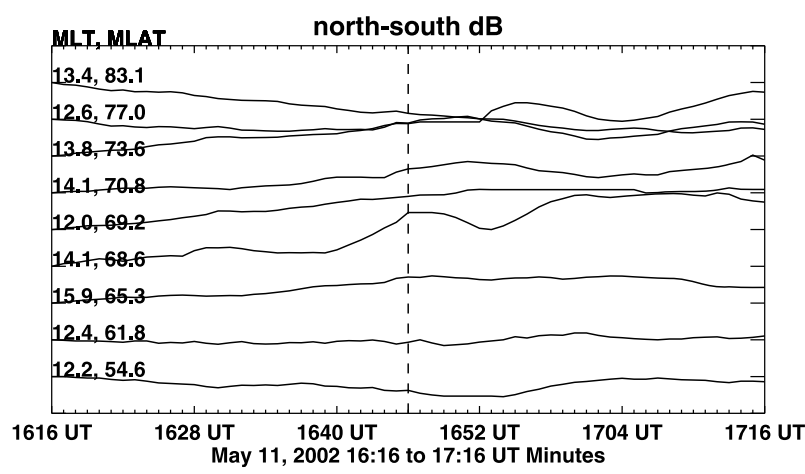

(a) Ground-based data

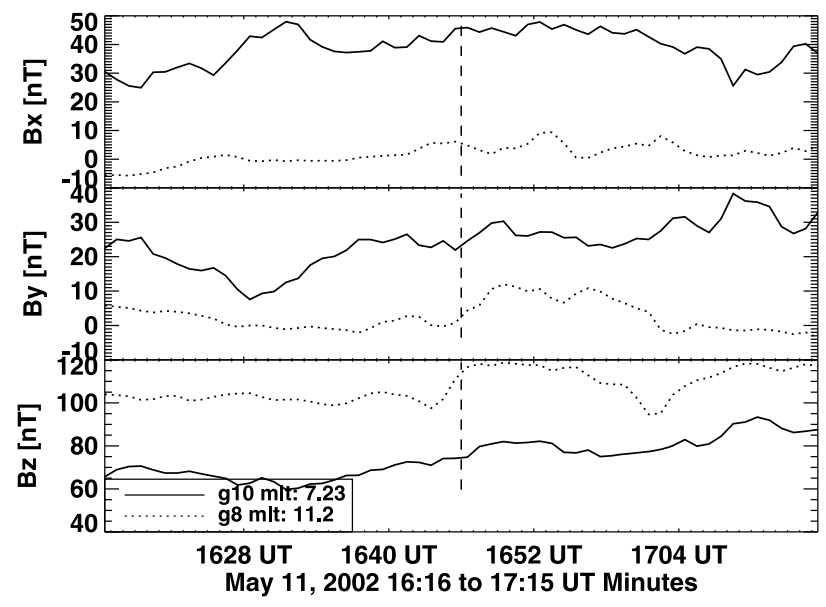

(b) GOES data

Figure 5. For the event on 11 May 2002, (a) the groundbased magnetic perturbations in the postnoon side and (b) magnetic field data from GOES.

situations, which therefore led us to classify these events into the "single-phase" group.

\section{Simulations With Different Alfvén Mach Numbers}

[22] While many parameters in the drivers could possibly control the properties of the response in the geospace system to solar wind density increases, as described above, only one parameter, the solar wind Alfvén Mach number, has been investigated here so far since the Mach number histogram indicates a direct possibility: a low Mach number may influence the responses to a sudden solar wind density increase. Examinations of the effect of other parameters on the system will be conducted in the future. The University of Michigan global MHD model Block-Adaptive Tree Solarwind Roe Upwind Scheme (BATSRUS) is used for this investigation [Powell et al., 1999; Ridley et al., 2004]. The MHD code has a block based structure in Cartesian coordinate, such that a wide range of scale sizes can be simulated in the same domain. A resolution of $1 / 4 R_{e}$ covers regions from $16 R_{e}$ on the dayside to $32 R_{e}$ on the nightside along the SunEarth line. The inner boundary of the MHD model is at 2.5 Earth radii, and is resolved with cell $1 / 8 R_{e}$ in size. This boundary is designed to have fixed density of $28 \mathrm{~cm}^{-3}$, floating thermal pressure and reflective velocity (i.e., no flow through the boundary). This MHD model is further coupled to the ionosphere, with the coupling technique described by Ridley et al. [2004]. No inner magnetosphere model is coupled to the MHD code in this study. Eleven simulations have been carried out with different Mach numbers. Mach numbers are varied through the IMF $B_{z}$ component, while keeping the density the same for the first serial of runs. Other simulations with Mach numbers that change through the density, as opposed to the IMF $B_{z}$ will also be performed and described later. Since the CPCP index and the ground magnetic perturbations are generally utilized to determine whether or not the system undergoes a two-phase response, section 3.1 will study the influence of the Mach number by primarily focusing on these two parameters. Also, as the two-phase response are phenomenologically similar under both southward and northward IMF conditions as found by Yu and Ridley [2009] (i.e., after the sudden increase in the solar wind density, the net changes in the ionospheric potential/FAC pattern are consistent), the following simulations only consider the northward IMF cases.

\subsection{Effect of the Mach Number (I) (Different IMF $B_{z}$ )}

[23] Eleven simulations with different Alfvén Mach numbers have been carried out. The solar wind and IMF conditions are initially modeled with constant IMF $B_{x}=$ $B_{y}=0, \mathrm{n}=5 \mathrm{~cm}^{-3}, V_{x}=400 \mathrm{~km} / \mathrm{s}$ and $\mathrm{T}=100,000 K$, but with different values of northward $B_{z}$ varying from 30 to $2.875 \mathrm{nT}$ in these eleven simulations. Therefore, the Alfvén Mach number varies from 1.3 to 14 . A simple contact discontinuity is then introduced at the upstream boundary at $32 R_{e}$, where the density and temperature abruptly change from $5 \mathrm{~cm}^{-3}$ to $20 \mathrm{~cm}^{-3}$ and from $100,000 \mathrm{~K}$ to $25,000 \mathrm{~K}$, respectively, in order to conserve the thermal pressure. The other parameters remain unchanged. Furthermore, the axis of the magnetic dipole is aligned with the rotation axis and the $\mathrm{Z}$ axis. Therefore, the simulations are idealized.

[24] The influence of the Mach number on the geospace system can be observed through the ionospheric cross polar cap potentials (CPCP). The CPCP in the simulation is the difference between the maximum and minimum of the electric potential over the ionospheric northern hemisphere. The CPCP for 11 different Alfvén Mach numbers are illustrated in Figure 9. Initially, the CPCP is steady before the high solar wind dynamic pressure encounters the bow shock. The different levels of the CPCP for these different runs are due to the fact that the IMF is becoming stronger as the Mach number is decreasing. This serves to increase the CPCP [e.g., Weimer, 1996]. After the sudden compression of the magnetosphere, the CPCP index with lower Mach numbers starts to increase earlier and shows three peaks through the simulation time, while the CPCP index with higher Mach numbers undergoes a two-peak behavior. The latter is consistent with the twophase responses in the ionosphere from early studies.

[25] To better serve the following discussions regarding the two-phase response in the CPCP index, the connection between the magnetospheric fast mode wave and the intensification of the ionospheric FAC or CPCP index as investigated by $Y u$ and Ridley [2009] is revisited. The Ey-response is generated by the fast mode wave propagating toward the Earth in the dayside magnetosphere. This fast mode wave induces dusk-to-dawn electric fields and currents that are connected with the duskward magnetopause current. Near the 
electric potential over electric potential
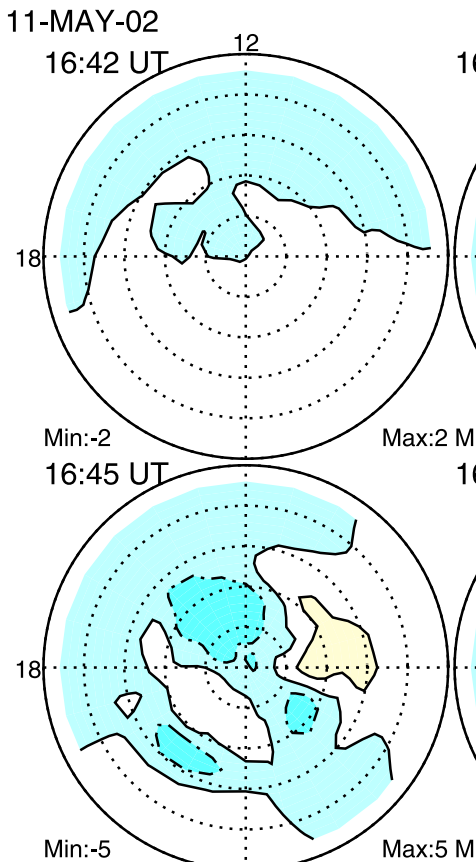

Min:-5
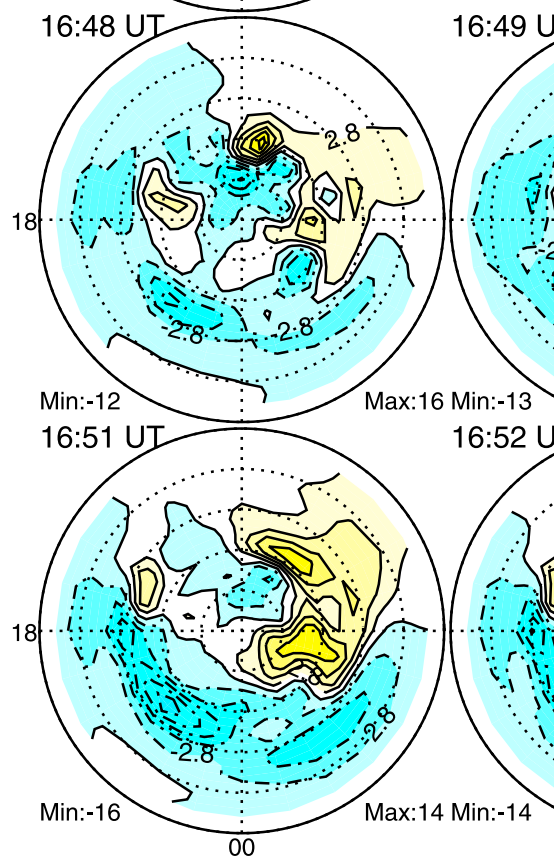

16:52 UT

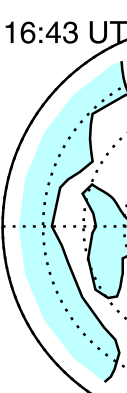

AMIE Version 2.21

Background time : $16: 42$ to $16: 44$ UT $16: 44$ UT $\quad 12$

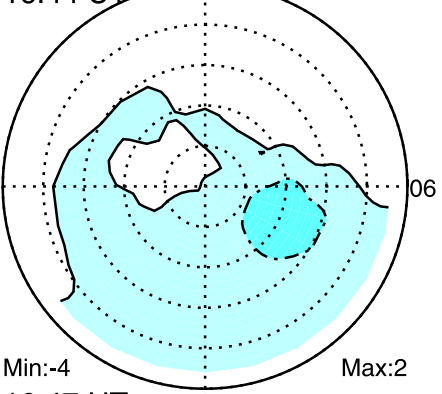

16:47 UT
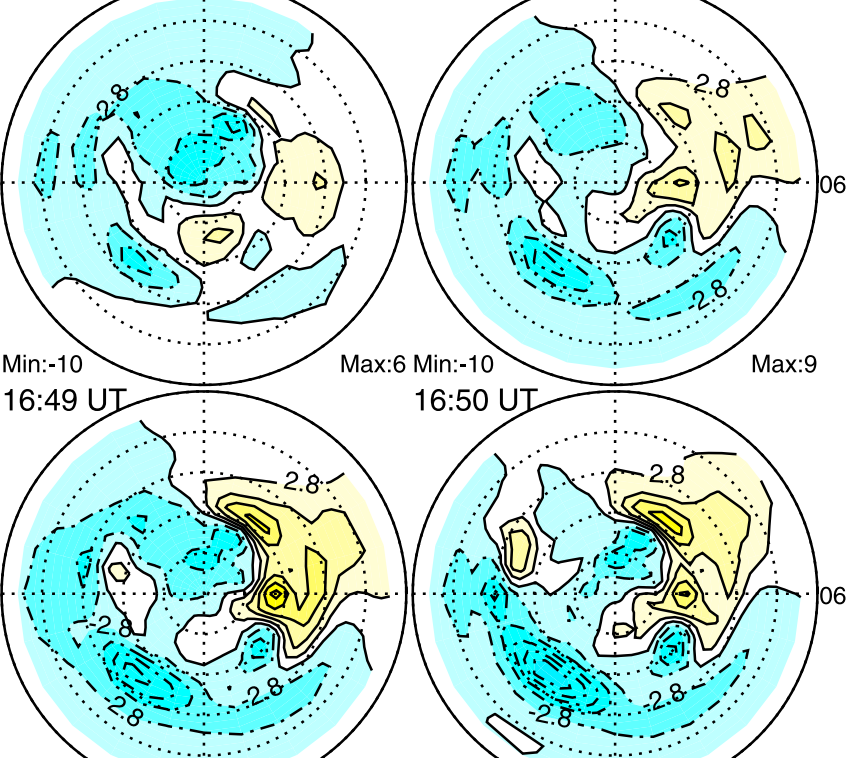

16:50 UT

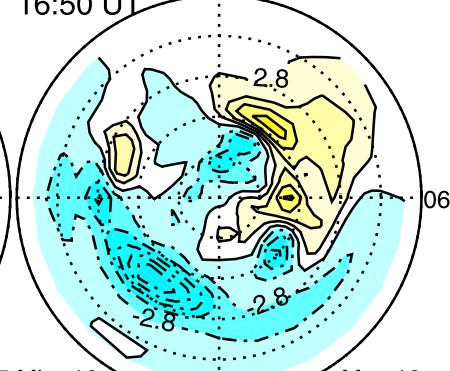

.

16:53 UT:-10

Max:13

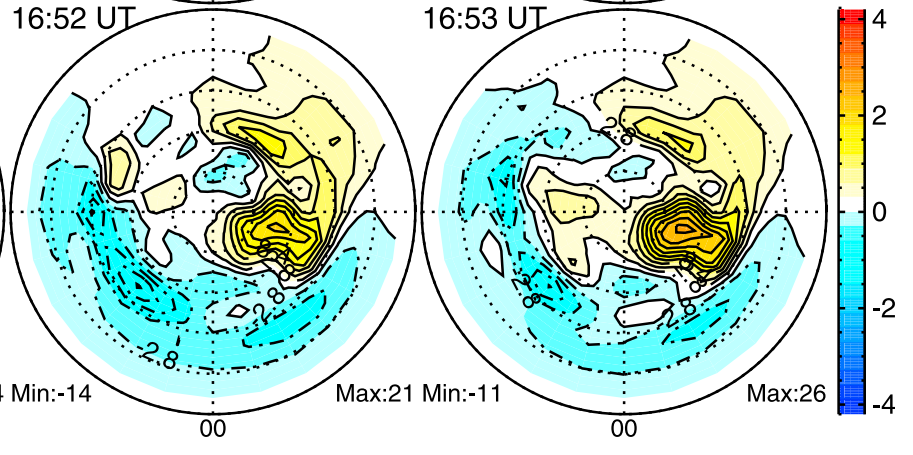

Figure 6. For the event on 11 May 2002, the residual electric potential obtained by subtracting the base potential pattern, an average of potential patterns from 16:42 to 16:44, from the other patterns. 

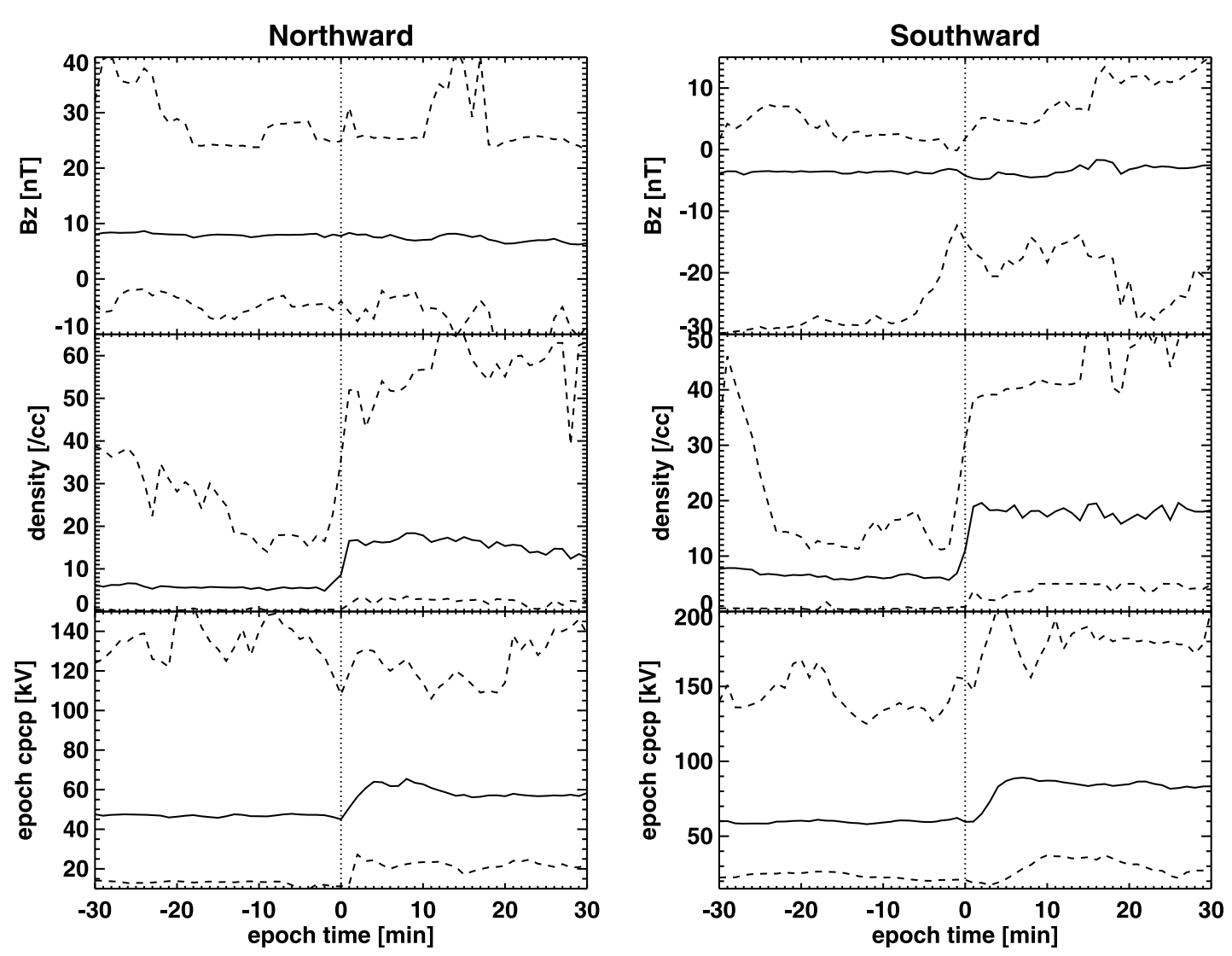

Figure 7. Superposed epoch plots of "two-phase" group for (left) northward and (right) southward IMF cases. The two dashed lines in each panel represent the upper and lower quartiles, and the dashed vertical line indicates the zero epoch time.

high-latitude magnetopause, this dusk-to-dawn current directs toward the ionosphere along magnetic field lines, subsequently disturbing the ionosphere. For the Vortexresponse (under northward IMF conditions), a bulge of high thermal pressure passes through the magnetosphere around the Earth creating two convection vortices in the dawn and dusk outer magnetosphere, generating FACs that connect to the ionosphere. These FACs are associated with the energy dynamo that propagates along with the bulge toward the nightside tail. These two FAC related phases are shown by $Y u$ and Ridley [2009, Figures 6 and 8].

[26] In the low Mach number simulations, the early response in the ionospheric $\mathrm{CPCP}$ can be interpreted as follows: A lower Mach number in the solar wind corresponds to a thicker magnetosheath, so the high-density discontinuity from the upstream boundary at $32 R_{e}$ encounters the bow shock earlier. Once the bow shock is compressed suddenly, a fast mode wave is triggered inside the magnetosheath ahead of the transmitted discontinuity, carrying the disturbance toward the magnetopause. This disturbance propagate inside the magnetosheath at a speed equal to the sum of the magnetosheath plasma speed $(\approx 100-200 \mathrm{~km} / \mathrm{s})$ and the fast mode wave speed $(\approx 300-400 \mathrm{~km} / \mathrm{s})$. However, in the high Mach number cases, the high-density discontinuity, still in the solar wind, has not yet encountered the bow shock and is propagating approximately at a solar wind speed, which is smaller than the above combined speed in the low Mach cases. For example, for a low Mach number of 2, the disturbances propagate inside the magnetosheath at about $500 \mathrm{~km} / \mathrm{s}$ because (1) the magnetosheath flow speed is not reduced significantly due to the weakness of the shock, and (2) the fast mode wave speed is large within the magnetosheath due to large magnetic fields. The discontinuity in a high Mach number of 12 would be still propagating in the undisturbed solar wind with a speed of $400 \mathrm{~km} / \mathrm{s}$. Therefore, the magnetopause is disturbed earlier in the low Mach number cases, producing earlier responses in the ionosphere, as observed in the CPCP index. It should be noted that, for a given event, the transmitted disturbance inside the magnetosheath propagates at a speed smaller than that when it was in the solar wind [e.g., Koval et al., 2006; Zhang et al., 2009; Pallocchia et al., 2010]. As contrasted with these references that studied the speed history in one given event, the discussion above is comparing different speeds between a low Mach number case and a high Mach number case.

[27] Also in the low Mach number simulations, the three peaks in the CPCP profile seem controversial because a two-peak profile is typical response and consistent with the two-phase responses. However, careful inspection of the ionospheric response indicates that the second peak in the CPCP profile is not from the Vortex-response (i.e., the second phase response); rather, it is still associated with the Ey-response (i.e., the first phase response). In other words, only the third peak is really caused by the Vortex-response. Take the simulation with Mach number 2.0 for example. After 17:28 UT, the Vortex-response takes over the entire 


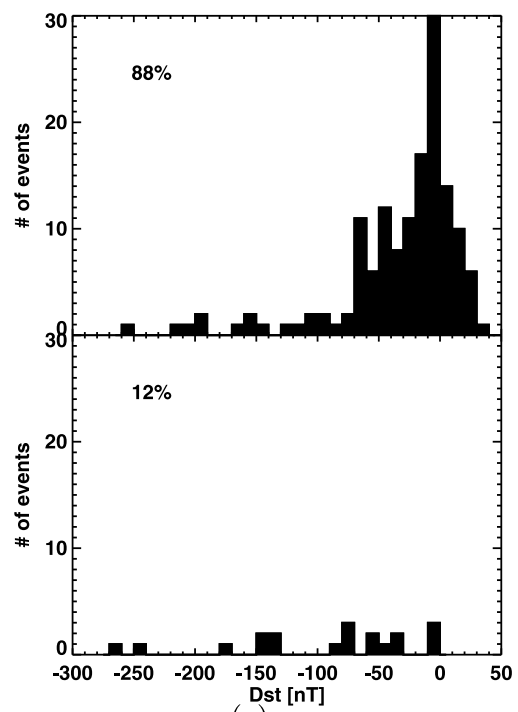

(a)

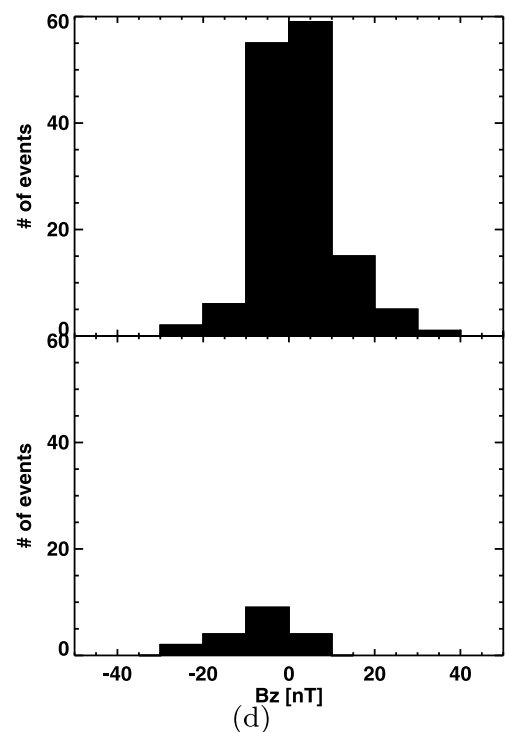

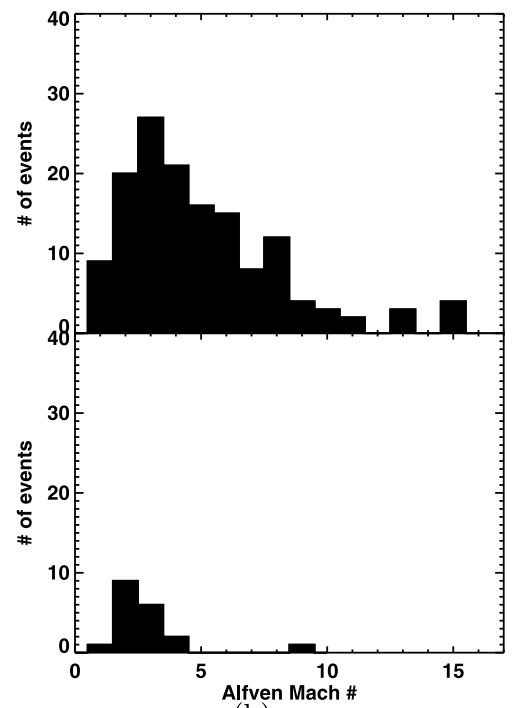

(b)

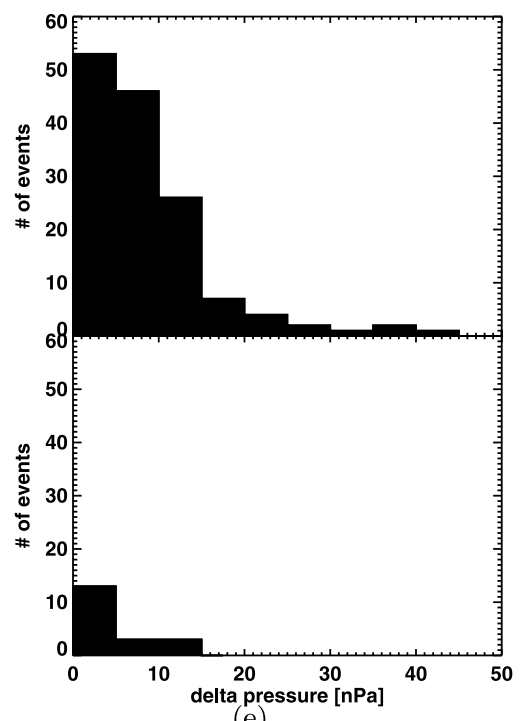

(e)

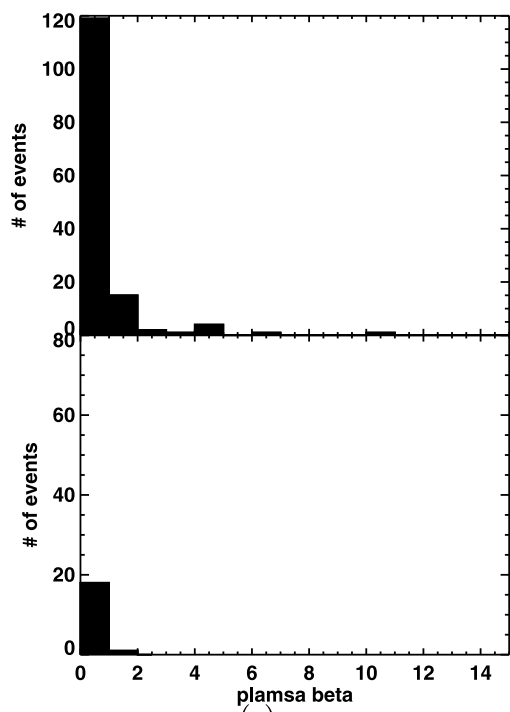

(c)

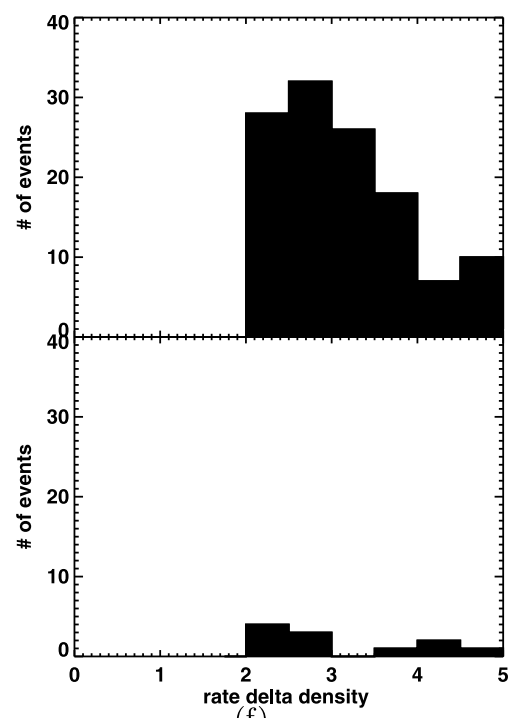

(f)

Figure 8. Histogram of events that show two-phase responses and single-phase response to the sudden solar wind density increase as a function of different factors: (a) $D_{s t}$ index, (b) Mach number, (c) solar wind proton $\beta$, (d) IMF $B_{z}$, (e) net dynamic pressure change, and (f) change rate solar wind number density. In each panel, the upper illustration refers to "two-phase" group, while the lower illustration refers to "single-phase" group. The percentages shown in the first panel stand for the relative numbers of (top) two-phase response and (bottom) single-phase response with respective to the total number of events.

ionospheric electrodynamics, but before this time, the electrodynamics on the ionosphere looks quite complicated. Figure 10 shows the residual field-aligned currents (FACs) and electric potentials. The first pair of FACs associated with the "Ey-response" (positive $J$ around 9 MLT and negative $J$ around 15 MLT) appears at 17:21:00 UT, and the second pair of FACs associated with the "Vortex-response" (positive $J$ around 15 MLT and negative $J$ around 9 MLT) emerges at 17:22:00 UT, while the first pair, together with the NBZ current system is still under growth. Subsequently, the positive electric potential cell on the duskside decreases a little bit (17:23:00-17:24:00) due to the appearance of the second pair of FACs (or the appearance of the Vortex-response) and increases again due to the continuous growth of the first
FAC pair (or the growth of the Ey-response), but the negative potential cell on the dawnside seems to continue increasing throughout the interval. The overall effect of these two different potential variations is that a subtle trough in the CPCP profile is produced.

[28] Since the CPCP index in these simulations is calculated as the difference between the maximum and minimum electric potential throughout the hemisphere, the decoupling of the CPCP profile could illustrate the above phenomena better. Figure 11 (top) shows the extremes (maximum and absolute value of the minimum) of the electric potential over the hemisphere. Similar to what is seen from the FAC and potential plots in Figure 10, during the early stage (17:21-17:28 UT, before the Vortex-response dominates 


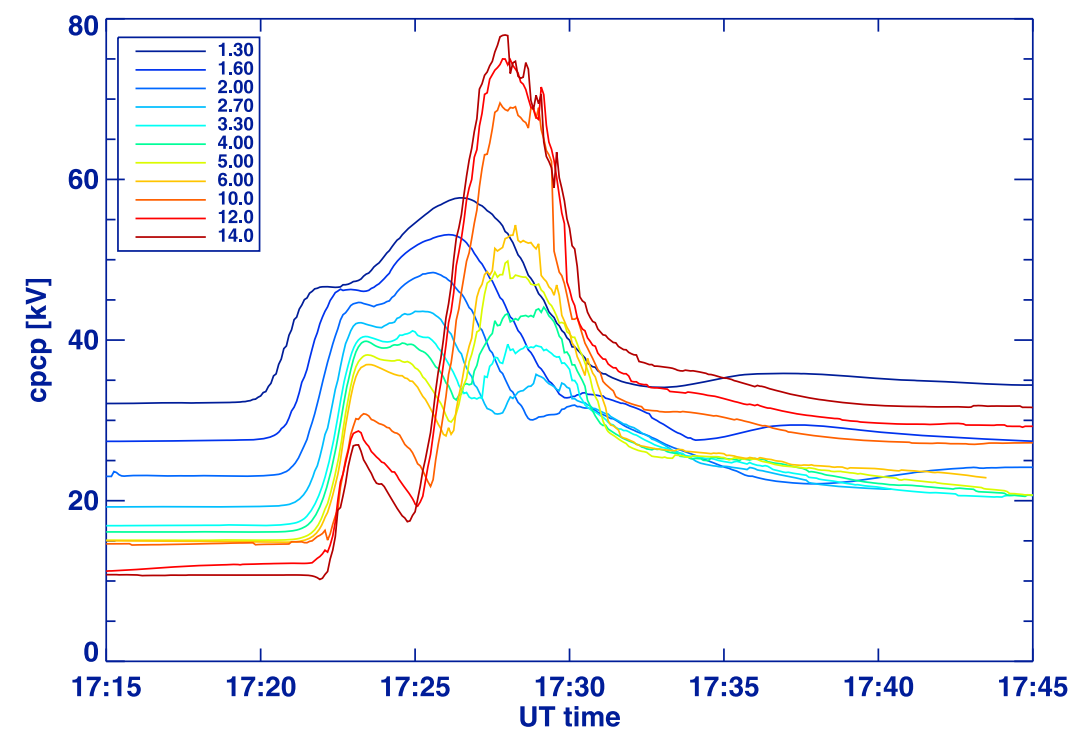

Figure 9. From idealized simulations, the cross polar cap potential reacts to the same solar wind density change from $N=5 \mathrm{~cm}^{-3}$ to $N=20 \mathrm{~cm}^{-3}$ with different solar wind Alfvén Mach number.

the ionospheric electrodynamics), the positive potential profile, representing the potential in the dusk cell, has two peaks, while the negative potential profile, representing the potential in the dawn cell in this stage, keeps growing until the Ey-response starts to fade. The reason for the double-peak in the positive potential is because the Ey-response is still in the growth mode, while the appearance of the Vortex-response, which has an opposite polarity and is initially much weaker than the Ey-response, cannot overwhelm the Ey-response, but only reduce its magnitude. Therefore, the two-peak profile is a result of the competition between the Ey-response and the initial Vortex-response. The increase of the negative potential without a secondary maxima indicates that the appearance of the Vortex-response on the dawn side does not influence the Ey-response as significantly as on the dusk side. Besides the different temporal evolution in the early stage of the dusk and dawn potential extremes, the reaction starting times from the two extremes also differs. The dusk (positive maximum) potential responds earlier than the dawn (negative minimum) potential.

[29] Given the idealized setup of the solar wind driving and the coordinates, these differences (in reaction time and potential variation) between the positive and negative potentials are likely to result from the conductance asymmetry between the dusk and dawn sides. To confirm this speculation, we repeated the simulation with Mach number 2.0, with a uniform conductance is applied on the ionosphere, instead of a more realistic conductance pattern that considers both the auroral oval and daylight. The Hall and Pedersen conductances here are replaced by constants 0 and $5 \mathrm{mho}$, respectively. The corresponding CPCP index from this simulation is shown in Figure 11 (bottom). It indicates that both negative and positive potential extremes react in the same manner to the sudden increase in the solar wind density: Both react at the same time and possess 3 peaks, with the subtle trough between the first two peaks resulting from the competition between the Ey-response and Vortex-response, as discussed above. The symmetric reactions in the positive/ negative extreme potentials develop because of the uniform ionospheric conductivity. Therefore, the distribution of the ionospheric conductivity is believed to play an important role in determining the asymmetry between the positive (dusk) or negative (dawn) potential after a sudden increase in the solar wind density arrives at the magnetosphere.

[30] Returning to Figure 9, in these low Mach number cases, the first two peaks are associated with the Ey-response that lasts longer, providing its early reaction time and late overtaking by the Vortex-response (after 17:26 UT in the high Mach number cases, and as late as 17:30 UT in the Mach number of 2.0 case). The third peak indicates the maximum strength of the Vortex-response, which is much weaker compared to those with high Mach numbers. This weaker Vortex-response in the low Mach number conditions is possibly due to the lack of energy input to the ionosphere. Kivelson and Ridley [2008] suggested that with a low Mach number and strong solar wind driving (high magnetic field), the efficiency of wave reflection in the ionosphere increases, leading to a reduced energy input into the geospace system. This suggestion was used to explain the saturation of the ionospheric CPCP and is more applicable for the relatively steady state, but not for the transient response as studied in this paper. Considering the response is associated with the FAC connecting with the magnetospheric vortices near the equatorial plane, the strength of the response has to be relevant to the dynamo $(\mathbf{J} \cdot \mathbf{E}<0)$ in the equatorial magnetosphere at this stage, which supplies the electromagnetic energy to the ionosphere via FACs. The dynamo is driven by the plasma flow from the thermal pressure gradient [Motoba et al., 2007] and is found to be significantly smaller than those with high Mach numbers (not shown). The smaller dynamo, or the smaller thermal pressure gradient after the sudden compression of the magnetosphere, implies that with a lower solar wind Alfvén Mach number, the energy through the magnetopause is reduced more significantly.

[31] Besides the above phenomena observed in the ionosphere, mainly from the CPCP index, the temporal variation of the ground magnetic perturbations also shows a strong dependence on the Alfvén Mach number. Figure 12 shows 

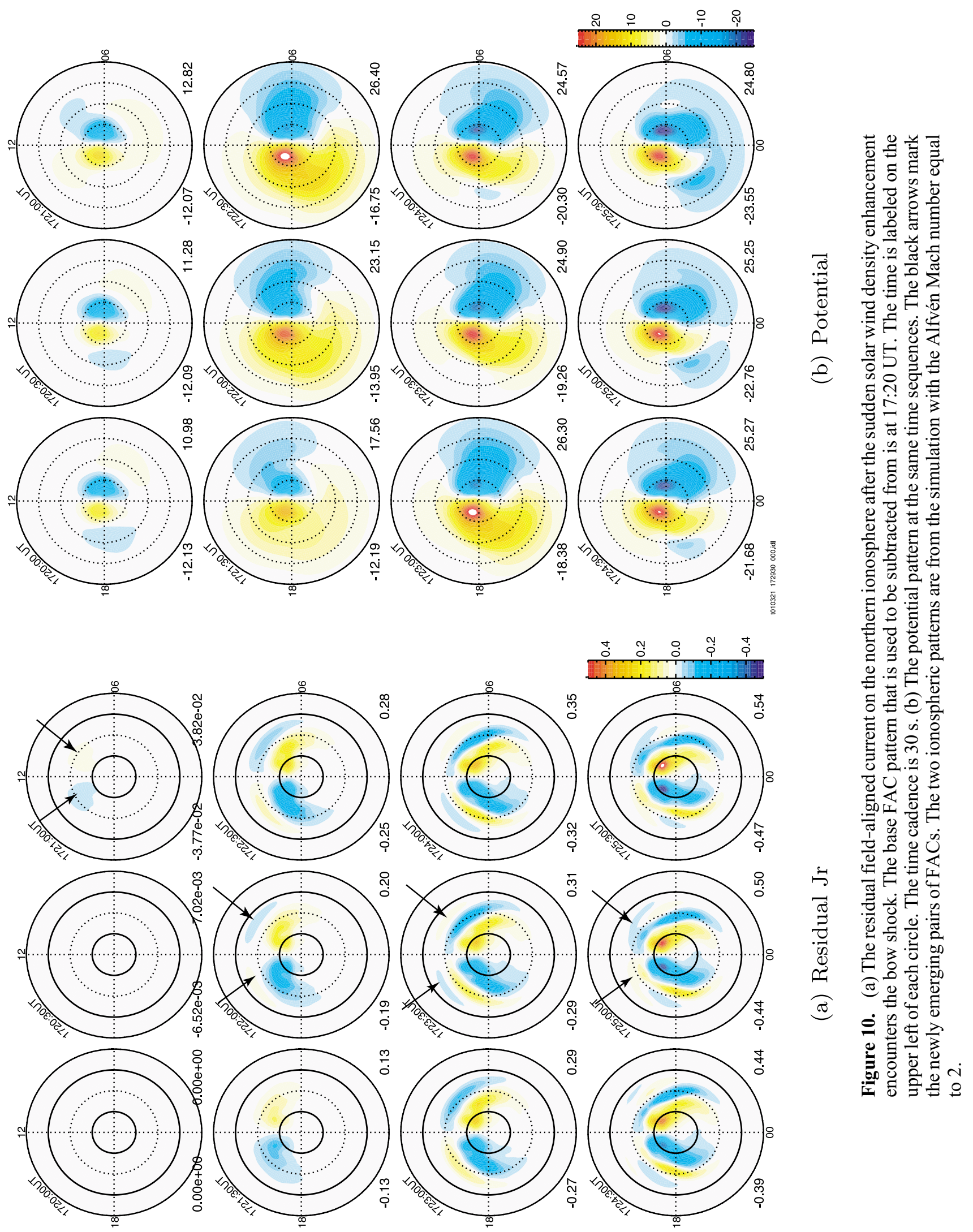

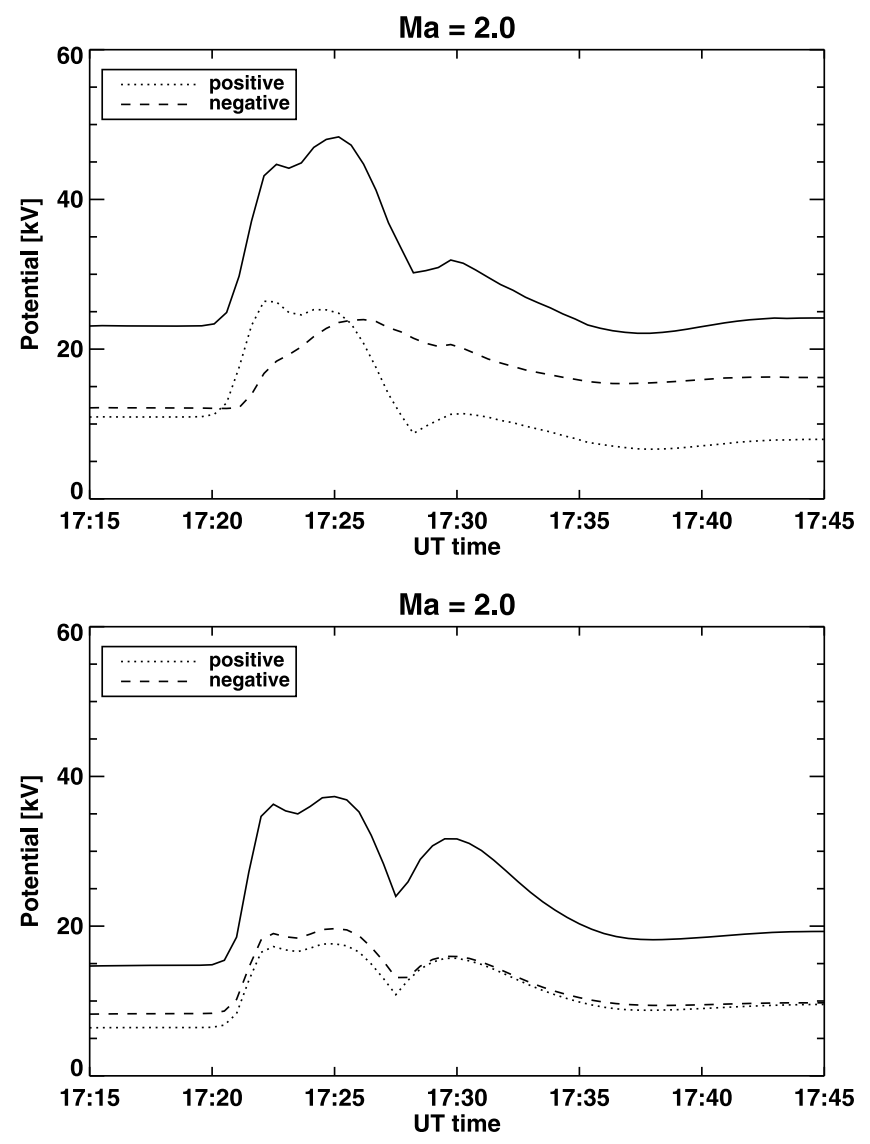

Figure 11. The CPCP index (solid), the maximum positive potential (dotted), and the absolute value of the minimum negative potential (dashed) in two simulations.(top) Simulation uses relatively realistic conductance, including the auroral oval and daylight. (bottom) Simulation uses uniform conductance, with the Hall and Pedersen conductance being 0 and 5 mho, respectively.

the northward magnetic perturbations at 15 MLT for Mach = 2.0 (Figure 12, top) and Mach $=14$ (Figure 12, bottom) at various magnetic latitudes. The perturbations are computed by Biot-Savart integral utilizing Hall currents above $50^{\circ}$ in the ionosphere. As the disturbances appear at high latitudes, the Hall current alone is sufficient to represent the groundbased perturbations [Yu et al., 2010]. The bipolar disturbances in both the Ey-response (i.e., positive disturbance at higher latitudes and negative at lower latitudes) and the Vortex-response (i.e., negative disturbance in higher latitudes and positive in lower latitudes) are clearly predicted in the high Mach number. But those with the low Mach number evolve in a similar complicated form as seen in the CPCP: the Ey-response experience two successive disturbances (most clear at $70^{\circ}$ and $75^{\circ}$ ), but the Vortex-response can be barely distinguished due to its weak strength.

\subsection{Effect of the Mach Number (II) (Different Densities)}

[32] In order to test the effect of the initial solar wind density on the magnetospheric response, the simulation suite was repeated but the conditions were altered. Instead of varying the IMF Bz component to obtain the different Alfvén
Mach numbers, the solar wind density was changed while keeping the IMF $B_{z}=5 \mathrm{nT}$. The initial solar wind density differs from one simulation to another (varying from 0.16 to $10 \mathrm{~cm}^{-3}$, which correspond to Mach numbers from 1.5 to 11.2), but the sudden density discontinuity is introduced in the same way in all the simulations: the density is increased by a factor of four. The CPCP index from these simulations is shown in Figure 13. The CPCP index with a lower Mach number again displays a three-peak form, but is significantly flatter than earlier simulations; while with a higher Mach number, the typical two-phase response is clear. This is consistent with the previous Mach number simulations and demonstrates that the solar wind Alfvén Mach number, either as a function of the density or IMF $B_{z}$, plays an important role in determining the temporal response in the system.

[33] Note that the Ey-response shown here is smaller for lower Mach numbers, which is contrary to what is shown in Figure 9 that the Ey-response in lower Mach number cases actually exceeds those in higher Mach number cases (i.e., the net increase of the Ey-response from the initial CPCP state is larger in lower number cases). This can be interpreted as follows. According to the earlier study by $Y u$ and Ridley [2009, Figure 6], the Ey-response in the ionosphere is related with FACs that are converted from the dusk-to-dawn
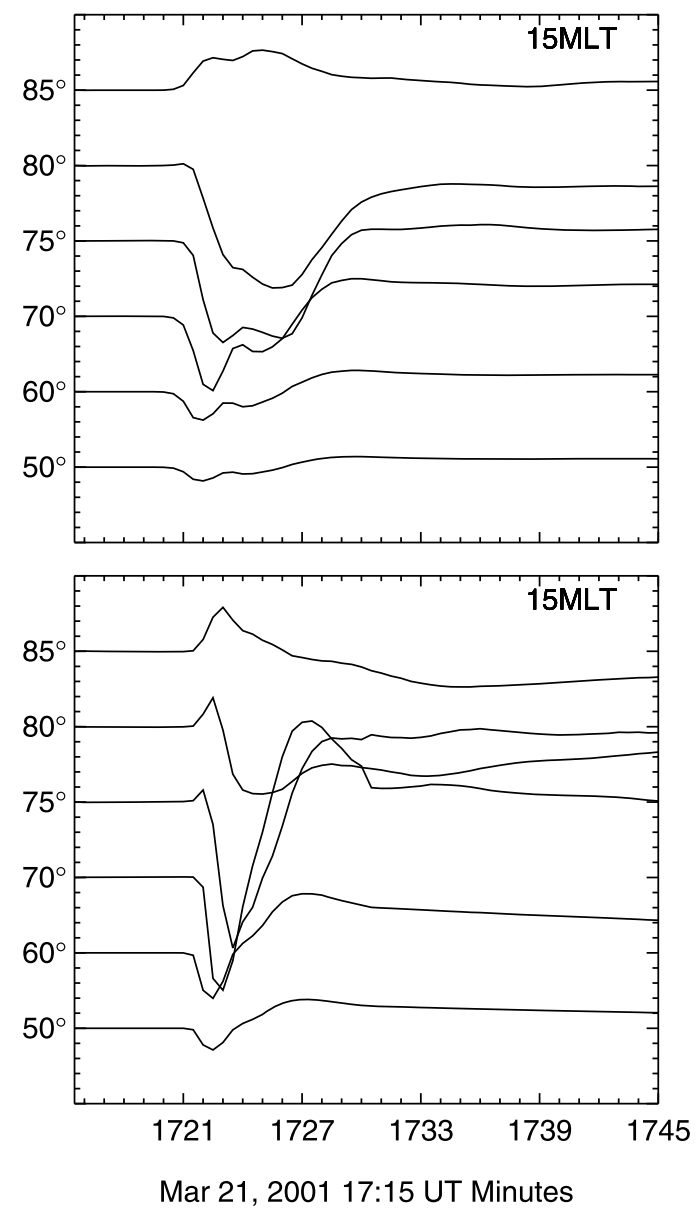

Figure 12. The northward magnetic perturbations at various latitudes along the 15 MLT longitude from two simulations: (top) Alfvén Mach number $=2.0$ and (bottom) Alfvén Mach number $=14.0$. 


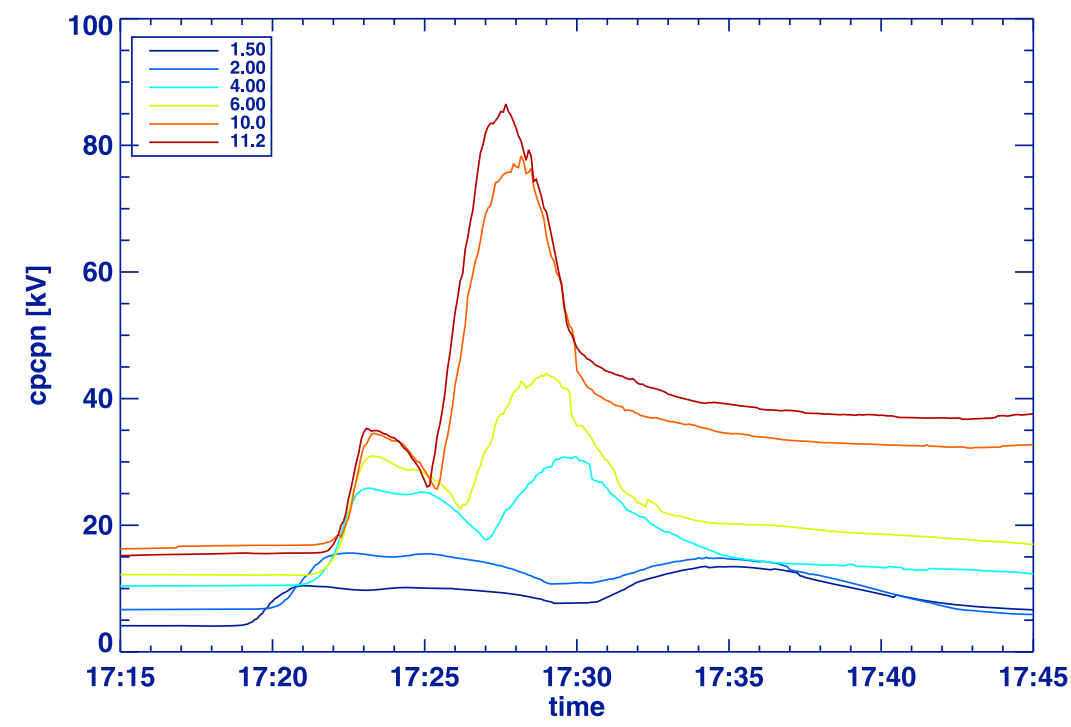

Figure 13. The CPCP from simulations with the same IMF $B_{z}$ but different solar wind densities. The sudden density discontinuity for these simulation undergoes the same jump ratio: by a factor of 4 .

inductive currents near the high-latitude magnetopause and are connected with the magnetopause currents, so the electric field near and at the magnetopause is an essential factor for the intensity of Ey-response. In Figure 9, larger Ey-response for lower Mach numbers is due to the larger $B_{z}$ in the solar wind and magnetosheath, which leads to larger electric fields near the magnetopause. In Figure 13, electric fields in the solar wind are the same due to the same IMF and solar wind velocity, but dissipation inside a thicker magnetosheath for a lower Mach number results in weaker electric fields at the magnetopause, thereby a less intense Ey-response in the ionosphere.

\subsection{Discussion}

[34] As shown in section 2, the responses of the ionospheremagnetosphere system to a sudden solar wind density increase can be categorized into two groups: "two-phase" and "singlephase". Within the "two-phase" response, the two phases are the Ey-response and Vortex-response. The "singlephase" group refer to the events that only show a single response, either with the Ey-response missing or the Vortexresponse missing, while the other group shows both of the responses in an orderly fashion. The statistical search for the causes of these two different classes was not conclusive due to the lack of statistics in the "single-phase" category. Additionally, the "two-phase" category does not indicate an explicit preference in any of the tested external solar wind parameters or the internal parameter $D_{s t}$.

[35] After performing simulations with various Mach numbers in an MHD model and inspecting the responses in the ionosphere (e.g., FAC and potential), we have been convinced that the two responses (Ey-response and Vortexresponse) always appear after a sudden solar wind density increase. It is found that the characteristics of the responses, including the duration, the strength, and especially the temporal variation of the response, highly depend on the Mach number. But it is differences in the response between the lower Mach number cases and the higher ones that make the recognizability of the typical two-phase responses difficult in the data. Indeed, in the low Mach number cases, the energy penetration into the system is significantly weakened, causing the lack of signal in the geosynchronous measurement (such as the GOES observations). Furthermore, the weak Vortexresponse and the long Ey-response as seen in the CPCP may also lead researchers to interpret that those events do not have the typical two-phase response. Indeed, as shown in the histogram for the Alfvén Mach number (Figure 8), almost all of the events (except one) selected for the "single-phase" category involve in relatively low Mach numbers. But as explored in the simulations, the two-phase response does exist after investigating the essential cause, the FACs; it is simply very weak and subtle. Hence, we propose that the two categories mentioned above actually belong to the same category. In other words, the second category ("singlephase") is the continuum of the first category ("two-phase").

\section{Wave Propagation}

[36] Of the eleven simulations with varying IMF $B_{z}$, the one with a nominal Mach number of 8 is chosen to examine the propagation of the compressional wave signal. In this case, the IMF $B_{z}$ is at a constant $5 \mathrm{nT}$. After the abrupt increase of density in the solar wind is introduced into the simulation domain at $32 R_{e}$, it subsequently encounters the bow shock and magnetopause, launching a compressional wave due to the sudden compression of the magnetosphere. The propagation of this wave signal can be examined by examining the temporal variation of the magnetic field inside and just outside of the magnetosphere after the sudden compression. Figure 14 (top left) shows the contour of $d B_{z} / d t$ from 3 to $15 R_{e}$ on the Sun-Earth line. Yellow means an increase of $\mathrm{Bz}$, and blue means a decrease of $B_{z}$. The roughly horizontal orange line overplotted on the contour can be interpreted as the position of the magnetopause, obtained by selecting the peak of the magnetopause current. The curves between the magnetopause and $2 R_{e}$ sketch the position of a compressional fast mode wave moving with the fast mode speed, launched at different locations/times, propagated through the MHD 

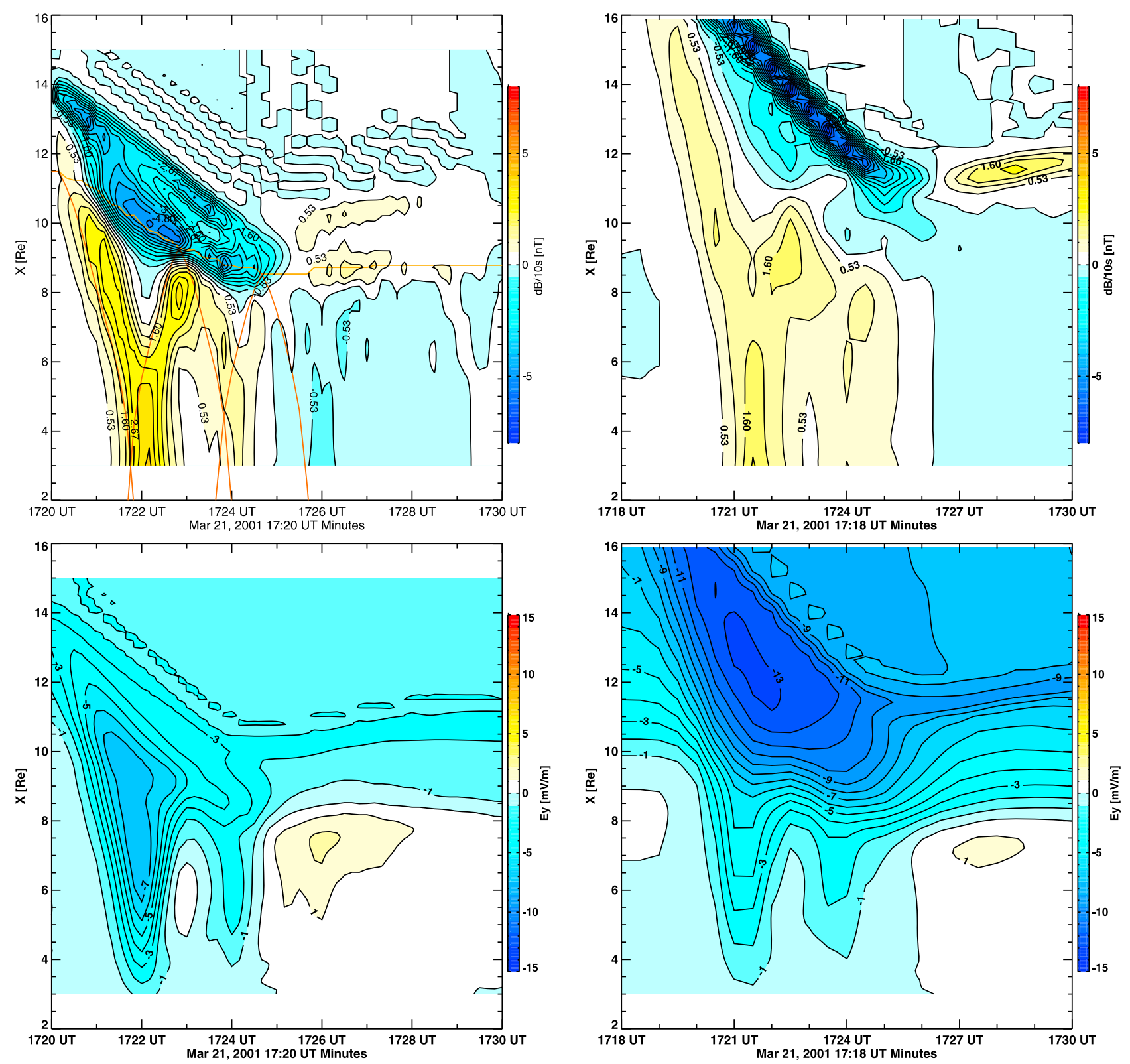

Figure 14. Results form two idealized simulations (Mach number $=8.0$ and 2.0): (top) the contour of $d B z / d t$ as a function of time on the Sun-Earth line from $3 R_{e}$ to $16 R_{e}$, with a Mach number of (top left) 8.0 and (top right) 2.0. (bottom) Same format but the color represents the duskward inductive electric field on the Sun-Earth line.

solution, given the magnetic field and density. Seen from Figure 14 , an increased $B_{z}$ (i.e., positive $d B_{z} / d t$ ) appears near the magnetopause at 17:20:00 UT and propagates toward the Earth at a fast mode wave speed, as the disturbance contour aligns with the fast mode wave curve. When the fast mode wave reaches close to the inner boundary of the simulation domain $\left(3 R_{e}\right)$, the wave is reflected back toward the magnetopause, where the wave is bounced back again toward the Earth at 17:23:00 UT. In other words, the compressional wave signal carried by the disturbance of $\mathrm{Bz}$ is reflected repetitively between the dayside magnetopause boundary and the inner boundary. Such bounces within the dayside magnetosphere cavity continue until the energy is dissipated. A similar behavior was reported by Samsonov et al. [2007] who also utilized a 3D global MHD model to demonstrate the wave propagation inside the magnetosheath and magnetosphere.

[37] In addition to the bouncing feature of the compressional wave signal propagation, another interesting phenomenon observed from the magnetopause profile is that the location of the magnetopause is well correlated with the above wave propagation. The magnetopause moves inward at a roughly constant rate until the reflected fast mode wave signal returns to the magnetopause (approximately 17:23 UT). The inward motion then slows significantly as the wave is reflected back toward the inner magnetosphere. When this wave returns (approximately 17:25 UT), the motion of the magnetopause actually reverses, and it is observed to move 


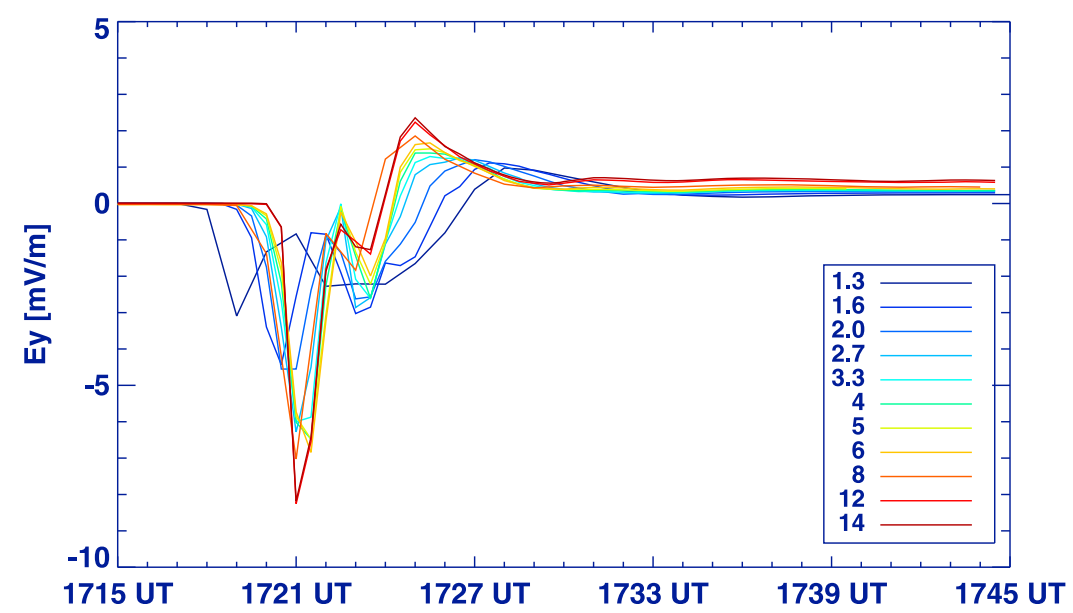

Figure 15. The duskward inductive electric fields at $7 R_{e}$ on the Sun-Earth line as a function of time, for different Mach number simulations.

away from the Earth (i.e., the magnetosphere expands). A few minutes later, the magnetopause motion ceases. Such motion of the magnetopause has also been studied by Samsonov et al. [2007]. Without numerical diffusion in the MHD code, this ringing of the magnetopause motion and bouncing of waves may have occurred for much longer.

[38] The above two features (i.e., the bouncing of the fast mode wave and the expansion of the magnetosphere subsequent to the sudden compression) are confirmed by the variation of the inductive electric field after the sudden compression. Figure 14 (bottom left) is the duskward inductive electric field $E_{y}$ on the Sun-Earth line from $3 R_{e}$ to $15 R_{e}$, caused by the sudden compression. Two waves of the large dusk-to-dawn electric field propagate toward the Earth after the compression, consistent with the two Earthward propagation of the fast mode waves from the magnetopause. Furthermore, a positive (dawn-to-dusk) electric field after 17:25:00 UT indicates a Sunward motion of the plasma, which is associated with the expansion of the magnetosphere subsequent to the anti-Sunward compression.

[39] The $d B_{z} / d t$ and the inductive electric field $E_{y}$ contours from a lower Mach number case $(\mathrm{Ma}=2.0)$ are also illustrated in the right column of Figure 14. Because of the difficulty in finding the magnetopause position based on the magnetopause current maximum in this case, the orange curves are not overplotted here. With a strong positive $B_{z}$ in the solar wind, there is little $d B_{z}$ across the magnetopause, and hence little current. An earlier start time is shown in the time axis, as the system reacts earlier than in the high Mach number case. But the vertical axis that represents the $\mathrm{X}$ position on the Sun-Earth line is the same, truncating portion of the thick magnetosheath during the early time period. Fortunately, the focus here is inside the magnetosphere. The wave reflection and the expansion of the magnetopause afterward are also clearly produced as in the high Mach number case. The differences in this case lie in the strength, the duration, and the depth into the magnetosphere of the wave penetration. It is observed that this low Mach number case is associated with weaker penetrations of $E_{y}$, which however lasts longer, into the inner magnetosphere (Figure 14, bottom right), even though the electric fields in the solar wind and the magne- tosheath are much stronger. Note that despite the weaker penetration of $E_{y}$ into the inner magnetosphere, the $E_{y}$ near the magnetopause, which is connected to the Ey-response in the ionosphere, has large magnitude, because of large $\mathrm{Bz}$ in the magnetosheath. This verifies that the Ey-response in the CPCP profile shown in Figure 9 is stronger for lower Mach numbers, as discussed in section 3.2.

[40] Figure 15 illustrates the dusk-to-dawn inductive electric field at $7 R_{e}$ on the Sun-Earth line within the dayside magnetosphere, as a function of time for different Mach numbers. The dusk-to-dawn electric fields (i.e., negative $E_{y}$ ) are significantly enhanced twice, seen in all of the simulations. This indicates that the propagation-reflection of the fast mode wave exists in all of these cases, no matter how small the Mach number is. The positive (duskward) peak, after the two strong increases of the dawnward electric fields, implies the expansion of the magnetosphere or the returning flow from the tail to the dayside. These are all consistent with the electric field contour plot in Figure 14. The final steady state in all of these cases is associated with positive (duskward) $E_{y}$, or Sunward plasma flow, which is larger than the initial condition (zero $E_{y}$ ). This implies that the impact from the sudden compression not only changes the magnetospheric convection configuration, but also the convection strength. The initial configuration is mostly dominated by the corotation convection, but after the transient response, the two-cell convection is stronger. This is consistent with Boudouridis et al. [2008], who reported from case studies of sudden increases in the solar wind density that the ionospheric convection is enhanced, compared to the initial state, after the transient increase subsides.

[41] Of the above features, the bouncing feature of the wave propagation has been partly verified by GOES satellite observations. Andréeová and Přech [2007] and Russell et al. [1999] have both shown two-step responses in the magnetic fields measured by GOES satellites orbiting inside the dayside magnetosphere when it was suddenly compressed by an interplanetary shock. The two-step response in the magnetic fields consists of an abrupt increase and a subsequent gradual increase. Samsonov et al. [2007] attributed this feature to the wave reflection as explored in their paper: the sharp increase 
in the magnetic field is due to the first Earthward fast mode wave and the gradual increase is caused by the reflected Sunward moving fast mode wave. While these GOES observations and corresponding interpretations provide evidence of the primary Earthward propagation of the fast mode wave and its reflection near the Earth boundary, the second Earthward propagation of the wave reflected from the magnetopause lacks observational verification yet. Samsonov et al. [2007] also predicted the multiple bounces of the fast wave in global MHD simulations, but no observational evidence for these waves other than the primary one has been reported in the literature. This requires further investigations in the future.

[42] Since the Ey-response in the ionosphere is related to the dayside magnetospheric dusk-to-dawn electric field $E_{y}$ induced from the sudden compression, the two enhancements of the dusk-to-dawn electric field should correspondingly cause the ionosphere to experience the Ey-response twice. In Figure 10, which illustrates the residual FACs in the ionosphere, the first Ey-response (upward, or yellow FAC around $10 \mathrm{MLT}$ and downward, or blue FAC around $14 \mathrm{MLT}$ at $70^{\circ}$ ) is seen at 17:21:00 UT while the second Ey-response of the same polarity appears at 17:23:30 UT, but at a lower latitude $64^{\circ}$ and with an elongated shape. The second FAC pair appears at a lower latitude because of the closer position of the magnetopause as it moves toward the Earth after the sudden compression. This low-latitude FAC pair propagates poleward as well as toward the nightside, and it fades away quickly and is found to have little influence on the potentials or the ground magnetic perturbations. Ionospheric observations have yet to report on this second Ey-response since it is so subtle, but our simulation predicts its existence. In that case, the response of the geospace system to the rapid increase of the solar wind density is no more two-phase, but at least three-phase: "Ey-response"-"Vortex-response"-"Eyresponse". The third response, however, is too weak to actually have effects on the ionospheric electrodynamics. It could be that ground magnetometers with better resolution at proper latitude and longitude positions in a clear-cut event may detect such a weak and fast variation on the ionosphere. Note that a forth pair of FACs emerges at 17:25:00 UT, resembling the Vortex-response, but, once again, has little influence on the system. This second Vortex-response indicates that the pair response (i.e., "Ey-response"-"Vortexresponse") may have to always be a pair. Therefore, our simulations predicts at least two such groups, leading to a four-phase response in the system: "Ey-response"-"Vortexresponse"-"Ey-response"-"Vortex-response". It is unclear whether there are more such two-phase responses subsequent to those. In our simulation so far, the wave amplitude at the dayside magnetosphere decreases significantly after two reflections, and hence two Ey-responses and two Vortexresponses are observed. Probably a stronger compression, which introduces a wave possessing stronger energy may allow the wave to reflect more times between the dayside magnetopause and the near-Earth boundary, could therefore produce more phases of the response. It should be noted that the ground-based observation (e.g., the magnetic field perturbation) or global activity index (e.g., CPCP) are not be able to reveal the second Ey-response or Vortex-response, given the fact that these responses are weak and more importantly appear at the time when the first Vortex-response is growing.

\section{Conclusion}

[43] This paper has studied the response of the geospace system to a sudden increase of the solar wind density by examining the observational data statistically and performing numerical simulations with different Mach numbers. We have reached the following conclusions:

[44] 1. While the ground-based observation suggests that the response to a sudden increase of the solar wind density can be generally categorized into two groups: "two-phase response" and "single-phase response" based on whether or not both an Ey-response and a Vortex-response turn up after the sudden compression of the magnetosphere, it is likely that the response may be a continuum, where one peak diminishes depending on the driver.

[45] 2. The solar wind Alfvén Mach number plays an important role in determining the temporal variation, the strength, and the duration of the response to the dynamic pressure increase. The Mach number alters the thickness of the magnetosheath and therefore the propagation characteristics of the discontinuity through the sheath. This leads the magnetosphere to react differently to similar density changes in the solar wind. In the lower Mach number case, the Ey-response starts earlier and lasts longer, which is then followed by a weaker Vortex-response.

[46] 3. In response to a sudden magnetospheric compression, a fast mode wave is launched. It propagates toward the Earth and is reflected back toward the magnetopause, where the wave is reflected back toward the Earth. The bouncing waves result in two enhancements of the dawnward inductive electric field in the dayside magnetosphere. The propagationreflection of the wave in the dayside magnetosphere appear to be associated with the inward and outward motion of the magnetopause.

[47] 4. Because the fast mode wave is reflected back and forth between the magnetopause and the inner boundary, a train of ionospheric responses are observed. The two-phase response is actually a four-phase response, where the second group are most likely caused by the reflected waves from the magnetopause, and may be too subtle to be observed in the ionosphere.

[48] Acknowledgments. We thank the OMNIWeb from NASA Goddard Space Flight Center for providing the solar wind observation data and the CDAWeb for providing the GOES satellite data. We also thank the following magnetometer data that was used: Carisma (Canadian Space Agency), IMAGE (Finnish Meteorological Institute), Measure (University of California Los Angles), Greenland coastal chains (Danish Meteorological Institute), MAGIC (University of Michigan), MACCS (Augsburg and Boston University), 210 magnetic meridian (Kyushu University and Nagoya University), and Intermagnet.

[49] Philippa Browning thanks Andrey Samsonov and another reviewer for their assistance in evaluating this paper.

\section{References}

Andréeová, K., and L. Přech (2007), Propagation of interplanetary shocks into the Earth magnetosphere, Adv. Space Res., 40, 1871-1880, doi:10.1016/j.asr.2007.04.079.

Araki, T. (1994), A physical model of the geomagnetic sudden commencement, in Solar Wind Sources of Magnetospheric Ultra-Low-Frequency Waves, Geophys. Monogr. Ser., vol. 81, edited by M. J. Engebretson, K. Takahashi, and M. Scholer, p. 183, AGU, Washington, D. C. 
Boudouridis, A., E. Zesta, L. R. Lyons, P. C. Anderson, and A. J. Ridley (2008), Temporal evolution of the transpolar potential after a sharp enhancement in solar wind dynamic pressure, Geophys. Res. Lett., 35 , L02101, doi:10.1029/2007GL031766.

Engebretson, M. J., et al. (1999), A multipoint determination of the propagation velocity of a sudden commencement across the polar ionosphere, J. Geophys. Res., 104, 22,433-22,452, doi:10.1029/1999JA900237.

Farrugia, C. J., M. P. Freeman, S. W. H. Cowley, D. J. Southwood, M. Lockwood, and A. Etemadi (1989), Pressure-driven magnetopause motions and attendant response on the ground, Planet. Space Sci., 37, 589-607, doi:10.1016/0032-0633(89)90099-8.

Friis-Christensen, E., M. A. McHenry, C. R. Clauer, and S. Vennerstrom (1988), Ionospheric traveling convection vorticies observed near the polar cleft: A triggered response to sudden changes in the solar wind, Geophys. Res. Lett., 15, 253-256.

Fujita, S., T. Tanaka, T. Kikuchi, K. Fujimoto, K. Hosokawa, and M. Itonaga (2003a), A numerical simulation of the geomagnetic sudden commencement: 1 . Generation of the field-aligned current associated with the preliminary impulse, J. Geophys. Res., 108(A12), 1416, doi:10.1029/2002JA009407.

Fujita, S., T. Tanaka, T. Kikuchi, K. Fujimoto, and M. Itonaga (2003b), A numerical simulation of the geomagnetic sudden commencement: 2. Plasma processes in the main impulse, J. Geophys. Res., 108(A12) 1417, doi:10.1029/2002JA009763.

Keller, K. A., M. Hesse, M. Kuznetsova, L. Rastätter, T. Moretto, T. I. Gombosi, and D. L. DeZeeuw (2002), Global MHD modeling of the impact of a solar wind pressure change, J. Geophys. Res., 107(A7), 1126, doi:10.1029/2001JA000060.

Kivelson, M. G., and A. J. Ridley (2008), Saturation of the polar cap potential: Inference from Alfvén wing arguments, J. Geophys. Res., 113, A05214, doi:10.1029/2007JA012302.

Kivelson, M. G., and D. J. Southwood (1991), Ionospheric traveling vortex generation by solar wind buffeting of the magnetosphere, J. Geophys. Res., 96, 1661-1667.

Koval, A., J. Šafránková, Z. Němeček, and L. Přech (2006), Propagation of interplanetary shocks through the solar wind and magnetosheath, $A d v$. Space Res., 38, 552-558, doi:10.1016/j.asr.2006.05.023.

Lukianova, R. (2003), Magnetospheric response to sudden changes in solar wind dynamic pressure inferred from polar cap index, J. Geophys. Res., 108(A12), 1428, doi:10.1029/2002JA009790.

Lysak, R. L., and D.-H. Lee (1992), Response of the dipole magnetosphere to pressure pulses, Geophys. Res. Lett., 19, 937-940.

Moretto, T., A. J. Ridley, M. J. Engebretson, and O. Rasmussen (2000), High-latitude ionospheric response to a sudden impulse event during northward IMF conditions, J. Geophys. Res., 105, 2521-2532, doi:10.1029/1999JA900475.

Motoba, T., S. Fujita, T. Kikuchi, and T. Tanaka (2007), Solar wind dynamic pressure forced oscillation of the magnetosphere-ionosphere coupling system: A numerical simulation of directly pressure-forced geomagnetic pulsations, J. Geophys. Res., 112, A11204, doi:10.1029/ 2006JA012193.

Pallocchia, G., A. A. Samsonov, M. B. Bavassano Cattaneo, M. F. Marcucci, H. Rème, C. M. Carr, and J. B. Cao (2010), Interplanetary shock transmitted into the Earth's magnetosheath: Cluster and Double Star observations, Ann. Geophys., 28, 1141-1156.

Powell, K. G., P. L. Roe, T. J. Linde, T. I. Gombosi, and D. L. D. Zeeuw (1999), A solution-adaptive upwind scheme for ideal magnetohydrodynamics, J. Comp. Phys., 154, 284-309.

Richmond, A. D., and Y. Kamide (1988), Mapping electrodynamic features of the high-latitude ionosphere from localized observations: Technique, J. Geophys. Res., 93, 5741-5759.

Ridley, A. J., and E. A. Kihn (2004), Polar cap index comparisons with AMIE cross polar cap potential, electric field, and polar cap area, Geophys. Res. Lett., 31, L07801, doi:10.1029/2003GL019113.

Ridley, A. J., T. I. Gombosi, and D. L. D. Zeeuw (2004), Ionospheric control of the magnetospheric configuration: Conductance, Ann. Geophys. $22,567-584$.
Russell, C. T., and M. Ginskey (1995), Sudden impulses at subauroral latitudes: Response for northward interplanetary magnetic field, J. Geophys. Res., 100, 23,695-23,702.

Russell, C. T., X. W. Zhou, P. J. Chi, H. Kawano, T. E. Moore, W. K. Peterson, J. B. Cladis, and H. J. Singer (1999), Sudden compression of the outer magnetosphere associated with an ionospheric mass ejection, Geophys. Res. Lett., 26, 2343-2346, doi:10.1029/1999GL900455.

Samsonov, A. A., D. G. Sibeck, and J. Imber (2007), MHD simulation for the interaction of an interplanetary shock with the Earth's magnetosphere, J. Geophys. Res., 112, A12220, doi:10.1029/2007JA012627.

Samsonov, A. A., D. G. Sibeck, and Y.-Q. Yu (2010), Transient changes in magnetospheric-ionospheric currents caused by the passage of an interplanetary shock: Northward interplanetary magnetic field case, J. Geophys. Res., 115, A05207, doi:10.1029/2009JA014751.

Sibeck, D. G., N. B. Trivedi, E. Zesta, R. B. Decker, H. J. Singer, A. Szabo, H. Tachihara, and J. Watermann (2003), Pressure-pulse interaction with the magnetosphere and ionosphere, J. Geophys. Res., 108(A2), 1095, doi:10.1029/2002JA009675.

Slinker, S. P., J. A. Fedder, W. J. Hughes, and J. G. Lyon (1999), Response of the ionosphere to a density pulse in the solar wind: Simulation of traveling convection vortices, Geophys. Res. Lett., 26, 3549-3552, doi:10.1029/1999GL010688.

Southwood, D. J., and M. G. Kivelson (1990), The magnetohydrodynamic response of the magnetospheric cavity to changes in solar wind pressure, J. Geophys. Res., 95, 2301-2309.

Stauning, P., and O. A. Troshichev (2008), Polar cap convection and $\mathrm{PC}$ index during sudden changes in solar wind dynamic pressure, J. Geophys. Res., 113, A08227, doi:10.1029/2007JA012783.

Tamao, T. (1964a), Hydromagnetic interpretation of geomagnetic SSC, Rep. Ionos. Space Res. Jpn., 18, 16-31.

Tamao, T. (1964b), The structure of three-dimensional hydromagnetic waves in a uniform cold plasma, J. Geomag. Geoelect., 18, 89-114.

Weimer, D. R. (1996), A flexible, IMF dependent model of high-latitude electric potential having "Space Weather" applications, Geophys. Res. Lett., 23, 2549-2552.

Yu, Y.-Q., and A. J. Ridley (2009), The response of the magnetosphereionosphere system to a sudden dynamic pressure enhancement under southward IMF conditions, Ann. Geophys., 27, 4391-4407.

Yu, Y.-Q., A. J. Ridley, D. T. Welling, and G. Toth (2010), Including gapregion field-aligned and magnetospheric currents in MHD calculation of ground-based magnetic field perturbations, J. Geophys. Res., 115, A08207, doi:10.1029/2009JA014869.

Zhang, H., Q.-G. Zong, D. G. Sibeck, T. A. Fritz, J. P. McFadden, K. Glassmeier, and D. Larson (2009), Dynamic motion of the bow shock and the magnetopause observed by THEMIS spacecraft, J. Geophys. Res., 114, A00C12, doi:10.1029/2008JA013488. (Printed 115(A1), 2010.)

Zhang, X. Y., et al. (2010), ULF waves excited by negative/positive solar wind dynamic pressure impulses at geosynchronous orbit, J. Geophys. Res., 115, A10221, doi:10.1029/2009JA015016.

Zong, Q.-G., X.-Z. Zhou, Y. F. Wang, X. Li, P. Song, D. N. Baker, T. A. Fritz, P. W. Daly, M. Dunlop, and A. Pedersen (2009), Energetic electron response to ULF waves induced by interplanetary shocks in the outer radiation belt, J. Geophys. Res., 114, A10204, doi:10.1029/ 2009JA014393.

Zong, Q.-G., B. W. Reinisch, P. Song, Y. Wei, and I. A. Galkin (2010), Dayside ionospheric response to the intense interplanetary shocks-solar wind discontinuities: Observations from the digisonde global ionospheric radio observatory, J. Geophys. Res., 115, A06304, doi:10.1029/ 2009JA014796.

A. J. Ridley and Y.-Q. Yu, Center for Space Environment Modeling, University of Michigan, 1417 Space Research Bldg., 2455 Hayward St., Ann Arbor, MI 48109, USA. (yiqunyu@umich.edu) 